\title{
Investigation of mode activity in NBI-heated experiments of Wendelstein $7-\mathrm{X}$
}

\author{
C. Slaby ${ }^{1}$, S. Äkäslompolo ${ }^{1,2}$, M. Borchardt ${ }^{1}$, J. Geiger ${ }^{1}$, R. Kleiber ${ }^{1}$, \\ A. Könies ${ }^{1}$, S. Bozhenkov ${ }^{1}$, C. Brandt ${ }^{1}$, A. Dinklage ${ }^{1}$, M. Dreval ${ }^{3}$, O. \\ Ford $^{1}$, G. Fuchert ${ }^{1}$, D. Hartmann ${ }^{1}$, M. Hirsch ${ }^{1}$, U. Höfel ${ }^{1}$, Z. Huang ${ }^{4}$, \\ P. McNeely ${ }^{1}$, N. Pablant ${ }^{5}$, K. Rahbarnia ${ }^{1}$, N. Rust ${ }^{1}$, J. Schilling ${ }^{1}$, A. \\ von Stechow ${ }^{1}$, H. Thomsen ${ }^{1}$, and the Wendelstein $7-\mathrm{X}$ team $^{5}$ \\ ${ }^{1}$ Max Planck Institute for Plasma Physics, Wendelsteinstr. 1, 17491 Greifswald, Germany \\ 2 Department of Applied Physics, Aalto University, FI-00076 Aalto, Finland \\ ${ }^{3}$ Kharkov Institute of Physics and Technology, 1, Akademicheskaya St., Kharkov, 61108, \\ Ukraine \\ ${ }^{4}$ MIT Plasma Science and Fusion Center, 167 Albany St, Cambridge, MA 02139, USA \\ ${ }^{5}$ Princeton Plasma Physics Laboratory, 100 Stellarator Rd, Princeton, NJ 08540, USA \\ E-mail: christoph.slaby@ipp.mpg.de
}

\begin{abstract}
The 2018 operation phase (OP 1.2b) of the stellarator Wendelstein 7-X (W7-X) included, for the first time, neutral beam injection (NBI) to heat the plasma. Since the injection geometry at W7-X is not parallel, this generates both passing and trapped fast particles. During longer phases of NBI injection, with the primary purpose to study the heating efficiency of this system, Alfvén eigenmodes (AEs) were observed by a number of diagnostics, including the phase contrast imaging (PCI) system, the magnetic pick-up coils (Mirnov coils), and the soft X-ray multi-camera tomography system (XMCTS).

Alfvén eigenmodes are of great interest for future fusion reactors as it has been shown that the resonant interaction of fast ions with self-excited AEs can lead to enhanced transport of fast ions and potentially to energy losses. This is especially true for so-called gap-modes, Alfvén eigenmodes with frequencies in gaps of the continuous spectrum, since they lack continuum damping. These modes are commonly known to be excited by fast ions, but other destabilizing mechanisms, e.g. the electron-pressure gradient are also possible.

In this article we present a first analysis of the experimentally observed frequencies from the theoretical side. The calculation of shear Alfvén wave continua for selected cases and the assignment of observed frequencies to the gaps of the continuous spectra are presented. Using the ideal-MHD code CKA [1], we find gap modes that match the experimental measurements in terms of the observed frequencies. We emphasize the crucial roles played by the coupling of sound and Alfvén waves as well as of the Doppler shift arising as a consequence of the radial electric field in W7-X.

We employ the perturbative gyrokinetic code CKA-EUTERPE [2], using a slowing-down distribution function for the fast ions as calculated by the Monte-Carlo particle following code ASCOT [3] to assess the fast-ion drive. We find that the fast-ion drive is insufficient to overcome the background-plasma damping. The fact that unstable modes were observed experimentally may point to problems with the modelling or indicate the existence of other destabilizing mechanisms, e.g. associated with the electron-pressure gradient [4] that sensitively depend on the profiles of the background plasma.
\end{abstract}

${ }^{5}$ see author list of T. Klinger et al., Nucl. Fusion 59112004 (2019) doi: 10.1088/1741-4326/ab03a7 


\section{Introduction}

The last operation phase of the optimized stellarator Wendelstein 7-X (W7-X), which was conducted from July till October 2018, was the first operation phase of this machine to feature neutral beam injection (NBI) and the generation of fast ions. The NBI system provides fast ions with much higher energies than those of the background-plasma ions. Hence, the fast ions are supposed to transfer that energy via collisions to the background plasma for heating purposes. Further, the NBI system also acts as a particle source and thus contributes to the fuelling of the plasma. While the fast ions transfer their energy to the background plasma, they slow down (i.e. thermalize) and their velocity may become resonant with the phase velocity (or fractions thereof) of Alfvén eigenmodes (AEs) in the plasma. Due to this resonant interaction the fast ions can transfer their energy to the Alfvén waves [5] which, in turn, grow in amplitude.

In general, AEs are an important issue for present-day devices as well as for future fusion reactors since these electromagnetic perturbations nonlinearly influence the particle trajectories through phase space and thus lead to enhanced transport, especially of the fast ions themselves [6]. This can reduce the performance of the fusion reactor. In general, the fast ions need to be well confined by the magnetic field of the device until they have slowed down in order to heat the plasma efficiently. This applies to fast ions generated by the heating systems (such as NBI, but also ion-cyclotron-resonance heating) and to fusion-born alpha particles equally. However, due to the nonlinear interaction with the Alfvén eigenmodes, the fast ions may be ejected from the plasma before they have thermalized. In that case, the consequences are energy losses and in extreme cases also damage to the first wall or plasma-facing components $[7,8]$. The damaging potential of NBI-generated fast ions could be observed during the last operation phase of W7-X when they supposedly melted a sample holder that was attached in a position of strong fastion losses on the first wall [9]. This problem had, at least in broad outline, been predicted theoretically a few years earlier. [10].

These issues illustrate the paramount importance of coming to a better understanding of the interaction of fast ions with AEs, especially in the complex three-dimensional magnetic fields of stellarators that provide more resonances between the fast ions and the Alfvén waves than the two-dimensional field in a tokamak does [11]. Note also that, in tokamaks, the control of AEs by means of applying external magnetic perturbations as well as controlled ECRH has already been demonstrated [12]. A stronger focus on stellarators is required if the stellarator option as a candidate for a future fusion reactor is to be pursued further.

Accordingly, in this paper we present the first theoretical investigation of Alfvénic mode activity that has been observed in the optimized stellarator W7-X during its last operation phase (denoted OP 1.2b). Related experimental findings from the same campaign have been reported and preliminarily analysed in [13]. We focus here on the modelling tools, our workflow and the current capabilities of the models. The emphasis of this investigation is on the possibility of fastion-driven modes, but we also explore the capabilities of the background plasma to destabilize the AEs observed experimentally. Note, however, that fast particles are an especially interesting issue for W7-X since this machine has been optimized for good fast-ion confinement at high beta (which remains to be shown experimentally) as well as MHD stability $[14,15]$. Further, the $55 \mathrm{keV}$ protons that the current NBI system supplies have the same normalized gyro-radius (normalized to the minor radius) as alpha particles in ITER. This enables W7-X to test if an optimized stellarator can confine the fusion products adequately in the absence of mode activity (neoclassical confinement). Once mode activity (due to the fast ions) is present the comparison to next-generation devices becomes more challenging as the distribution function of the fast ions is expected to differ. This will lead to a different spectrum of modes that are excited and will change the associated nonlinear fast-ion transport. Nevertheless, this does not diminish the importance of fast-ion studies in present-day devices.

$\mathrm{W} 7-\mathrm{X}$ is an optimized superconducting stellarator with a major radius $R=5.5 \mathrm{~m}$ and a minor 


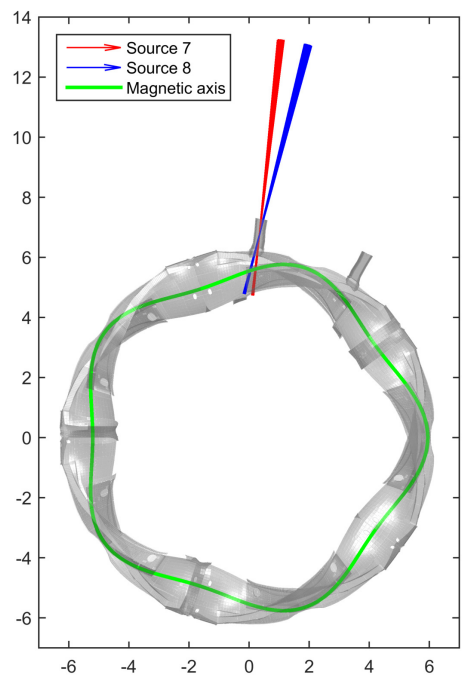

Figure 1: Schematic top-down view of Wendelstein 7-X showing also the injection geometry of the two NBI sources available in the last campaign. The second NBI box (its beam duct is already visible in the figure) will be commissioned in the next operation phase. The green line indicates the magnetic axis.

radius $a \cong 0.5 \mathrm{~m}[16]$ (depending on the magnetic configuration). Ref. [16] also provides a good overview of operational parameters and milestones that have been reached in the last campaign. A schematic view of W7-X, nicely showing the five-fold symmetry of the device, is shown in Fig. 1. The figure also illustrates the injection geometry of the NBI system showing the two sources of the single beam box that was available during the last campaign. It is easy to see that the NBI system at W7-X injects fast particles quite perpendicularly into the plasma. Therefore, both passing and trapped fast ions are generated, which can then excite magneto-hydrodynamic (MHD) modes. For instance, helically trapped fast ions have been shown to excite resisitive interchange modes in LHD plasmas with strong perpendicular NBI [17]. In DIII-D, a mix of parallel and perpendicular NBI has been used recently to study how fast ions interact with tearing modes [18]. The NBI geometry at W7-X is taken into account in the calculation of the fast-ion distribution function.

During OP $1.2 \mathrm{~b}$, MHD mode activity was observed in W7-X by a number of diagnostics in many discharges. This applies to discharges that were exclusively heated by electron-cyclotronresonance heating as well as to ones that included NBI. This suggests that not only the fast ions, but also the background plasma is capable of exciting AEs in W7-X. Also from the previous operation phase OP 1.1, there exist observations of Alfvénic activity that was possibly excited by the free energy of the electron-pressure gradient [4] in the absence of fast particles. We will continue to explore the possibility of modes driven by the background plasma in this article by choosing the appropriate kinetic particle species in our CKA-EUTERPE modelling.

The wealth of experimental data that was gathered in the last operation phase requires dedicated and powerful tools for data evaluation, specifically for the generation of spectrograms and to extract mode structures and mode numbers from the data. Recently, such tools (which already existed for other problems in mathematics, physics, or civil engineering) have been adapted for applications in plasma physics [19]. We present here the application of the DMusic algorithm $[20,21]$, a powerful tool to generate spectrograms, to our experimental data. This tool provides a much better visibility of the signal in the frequency domain than an FFT-based method would. Another algorithm from stochastic systems identification (SSI for short) $[22,23]$ is used to extract poloidal mode numbers from an array of Mirnov coils. Both the DMusic and the SSI algorithms are described in detail in the references given above, but another forthcoming 
article, Ref. [19], details and summarizes their strengths for analysing data from plasma physics, be it experimental or simulation data.

Thus, the goals of this article can be summarized as follows: We first show the progress of the data evaluation tools and how they are applied to experimental data from the last campaign. This is done for the example of a specific NBI-heated discharge in which Alfvénic mode activity at different frequencies was present. This discharge is not necessarily representative of the 'typical' discharge from OP 1.2b. We use it as an example to illustrate our workflow, but also as a first test to try to offer a theoretical explanation of the experimentally observed mode activity. In this sense, this paper reports on the first validation efforts of our numerical tools that calculate the shear Alfvén continuum, the Alfvén eigenmodes and their kinetic destabilization with actual W7-X data. However, as this is the first attempt of this complex task for W7-X, a number of challenges remain for the future. While the calculation of the shear Alfvén continua and the assignment of the observed modes to gaps in the spectra works well, the gyrokinetic CKAEUTERPE modelling can at the moment not explain why any unstable modes were observed experimentally. Nevertheless, we want to follow the approach of using consistent input data as close to the experimental measurements as possible - throughout the analysis chain. Only in this way can we identify the parts of our analysis that work and find out which steps need to be improved. The discrepancy between the CKA-EUTERPE results and the measurements could be due to a number of reasons that are discussed in this article. A detailed discussion of the future challenges will be given in the summary section.

The outline of this paper is as follows: In Sec. 2 we start by introducing our modelling codes as well as our tools for data evaluation. Sec. 3 highlights our experimental measurements for one discharge from the last campaign. Shear-Alfvén continua for this case are shown in Sec. 4, where we also discuss what effects the coupling to sound waves and the presence of a radial electric field have on the continua. The experimentally observed frequencies can be assigned to gaps in the continua. Guided by the analysis of the experimental data, we use the CKA code in Sec. 5 in order to find Alfvén eigenmodes in the frequency gaps of the continuous spectra. The kinetic drive of these modes with respect to different kinetic species (including also the fast ions) is discussed in Secs. 6 and 7. Finally, conclusions are drawn in Sec. 8.

\section{Analysis tools}

The aim of this article is to present our modelling tools and to apply them to a specific case of measured mode activity from the last campaign. We aim to identify the observed modes in terms of their poloidal and toroidal mode numbers and radial location. We further want to quantify the ability of the fast ions as well as the background plasma to drive these modes unstable. For this complex task a number of numerical tools are used.

The DMusic algorithm $[20,21]$ is used to calculate spectrograms that allow us to identify modes in the phase contrast imaging (PCI), soft X-ray multi-camera tomography system (XMCTS) [24], and Mirnov measurements. The SSI tool (stochastic system identification) $[22,23]$ can also detect common frequencies in an array of time signals and extract poloidal mode numbers. Shear Alfvén continua are computed by the CONTI [25] code. Given the knowledge of the location of the frequency gaps, we can use CKA [1] to find discrete eigenmodes in these gaps. As has already been demonstrated in the past [26-28], we can finally use CKA-EUTERPE [2], the perturbative version of the gyrokinetic, three-dimensional, electromagnetic particle-incell code EUTERPE, to infer the power transfer between a kinetic particle species and these pre-computed modes. There are a number of reasons why the perturbative model, which lacks the fidelity of a fully gyrokinetic approach, has been been chosen in this work: CKA-EUTERPE is much faster than a fully gyrokinetic model, which is important, because the stability of a large number of possible modes needs to be investigated (see Sec. 7). A fully gyrokinetic model would be impractical for this task. Further, the perturbative model is numerically more stable 
and allows us to easily separate the different kinetic species to calculate damping and drive independently. This would not be as easily possible with a higher-fidelity gyrokinetic model. Note, however, that the present investigation can still be used as a starting point for more advanced fully gyrokinetic simulations in the future.

In order to assess the fast-ion drive, we need the fast-ion distribution function in energy space as well as their radial density profile. Both is supplied by the ASCOT code [3].

Since most of these tools have been extensively described and verified in the past, we will not describe them in detail here. Also, distribution functions computed by ASCOT have been used in CKA-EUTERPE simulations before $[27,29]$ (albeit not for a real W7-X experimental programme).

However, since now real experimental data are the starting point of this investigation, their proper analysis is an important aspect of this work. The DMusic algorithm and the SSI tool are used to compute eigenmode frequencies and poloidal mode structures. Since this is one of the first times that these tools are applied to data measured at W7-X, they deserve to be briefly described here. Nevertheless, we would like to refer the reader to Ref. [19] which gives a more in-depth account of the mathematical foundations of these algorithms.

\subsection{The DMusic method}

The DMusic algorithm (used for the calculation of the spectrograms in this article) is a parametric method that, in contrast to non-parametric methods (e. g. a Fast Fourier Transform) leads to a better frequency resolution and to an improved handling of noisy signals. Two important parameters that determine the appearance of the spectrogram are $J$ and $K$ (see below). In practice, they can be chosen in a way to shift the focus of the method to a specific feature of interest (i.e. a certain mode). Thus, the DMusic algorithm is best used in connection with a traditional FFT - the FFT provides an overview and identifies interesting features which then can be investigated with DMusic in more detail. At the core of the method is a linear prediction ansatz

$$
y(n)=\sum_{i=1}^{J} c_{J-i} y(n-i)
$$

for the discrete signal $y$ of length $N$. Applying this ansatz to the last $N-J$ values of $y$ gives a linear system for the coefficients $c_{0}, \ldots, c_{J-1}$ featuring a Hankel matrix $A$. In the DMusic algorithm one makes use of the fact that a matrix corresponding to $A$ (usually denoted by $\hat{A}$ ) can be calculated by assuming a signal model

$$
\hat{y}(n)=\sum_{i=1}^{K} a_{i} \exp \left[s_{i} n\right]+w(n)
$$

with white noise $w(n)$ and complex frequencies $s_{i}$ (to be determined). Using the properties of $\hat{A}$ as a Hankel matrix, it can be shown analytically that this matrix can be decomposed into parts that lie in the so-called signal and noise spaces, respectively. These two spaces are orthogonal to one another. One thus varies the $s_{i}$ until the singular vectors of $A$ (determined with an SVD) are orthogonal to the noise space. More details and the application of this method to gyrokinetic simulations are shown in Ref. [19].

\subsection{The SSI method}

The idea behind the SSI method (a detailed description of which can also be found in Refs. [19, 30]) is the following: We have a linear system

$$
\begin{aligned}
\mathbf{x}_{k+1} & =A \mathbf{x}_{k}+\mathbf{w}_{k} \\
\mathbf{y}_{k} & =C \mathbf{x}_{k}+\mathbf{v}_{k}
\end{aligned}
$$




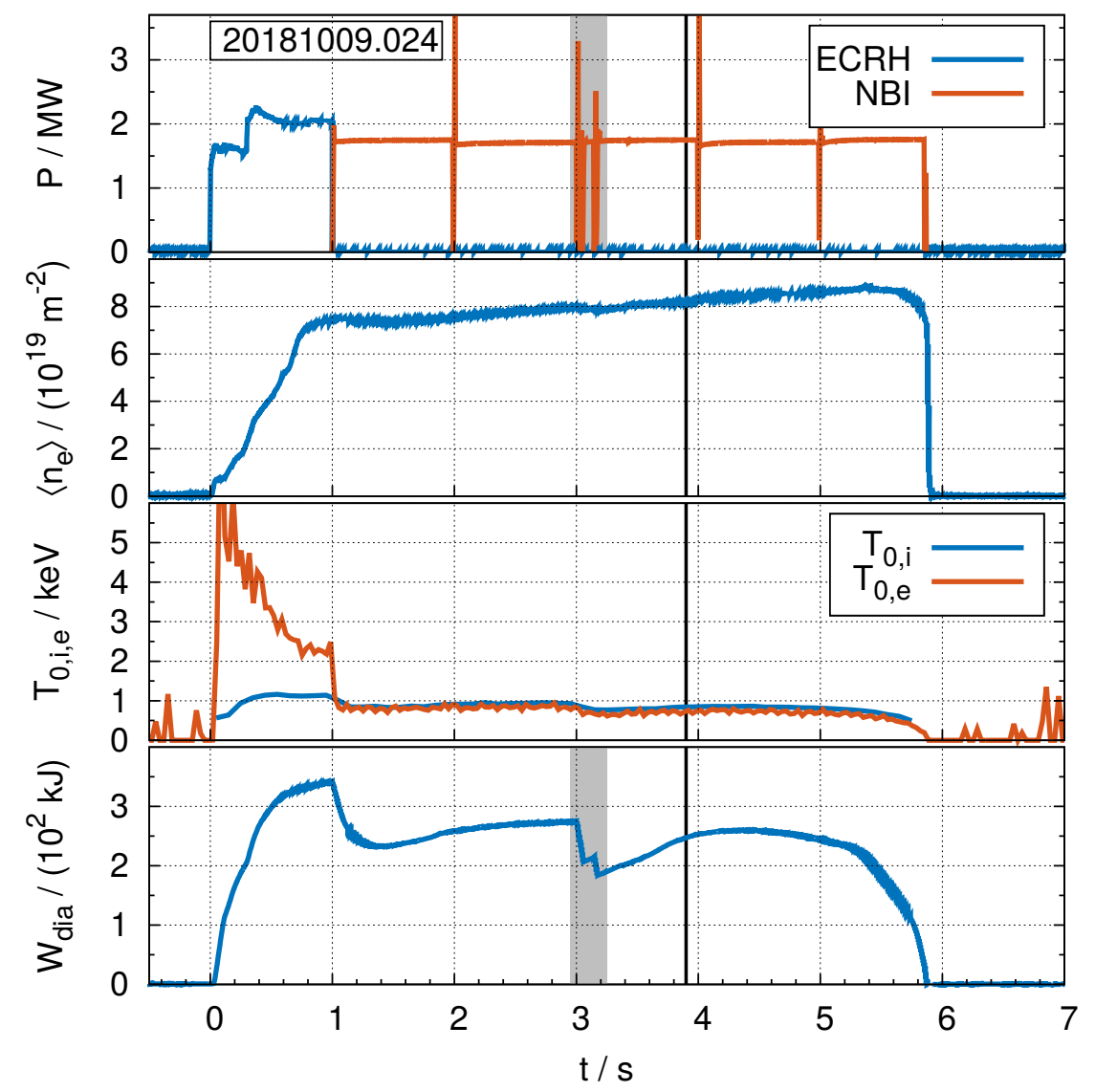

Figure 2: Time traces of key quantities in discharge 20181009.024. From top to bottom: Injected power, line-averaged electron density, central ion and electron temperatures, and diamagnetic energy. The discharge terminated due to increasing radiation and low diamagnetic energy at $t=5.9 \mathrm{~s}$. The time at which the mode analysis of this paper will be performed is indicated by the vertical black line at $t=3.9 \mathrm{~s}$. Some modes are first excited by the switching of the NBI sources at around $t \approx 3.0 \mathrm{~s}$. During this time also the diamagnetic energy drops (see gray-shaded region).

with the following meaning: $k$ measures (discrete) time, and $\mathbf{y}_{k}$ is an indirect observation made by some measurement system, e. g. a system of poloidally separated Mirnov coils. The dimension of the vector $\mathbf{y}$ is equal to the measurement channels of the diagnostics. The observation made by the diagnostics is related to $\mathbf{x}_{k}$, but also includes white noise $\mathbf{v}_{k}$, which is, for instance, due to the measurement process. The quantities of interest are, however, the $\mathbf{x}_{k}$, which are linearly related to the observation and driven by white noise $\mathbf{w}_{k}$. The meaning of the $\mathbf{x}_{k}$ is that of a 'hidden dynamical system'. One finds that the eigenfrequencies of the system are the eigenvalues of $A$ (which cannot be directly observed), and the eigenfunctions are found when the matrix $C$ is applied to the eigenvectors of $A$. Thus, the challenge is to determine the matrices $A$ and $C$ from the observations. The procedure is implemented algorithmically by the SSI method.

\section{Experimental measurements and data analysis}

We aim to analyse W7-X discharge 20181009.024 conducted in October 2018 (during operation phase OP $1.2 \mathrm{~b}$ ). This discharge was part of the NBI heating experiments performed to quantify the performance of this particular heating system. As such, it featured some of the longer NBI pulses in OP $1.2 \mathrm{~b}$. The time traces of heating power, line-averaged density and temperature as well as stored energy are shown in Fig. 2. As can be seen in the top panel, the initial plasma 

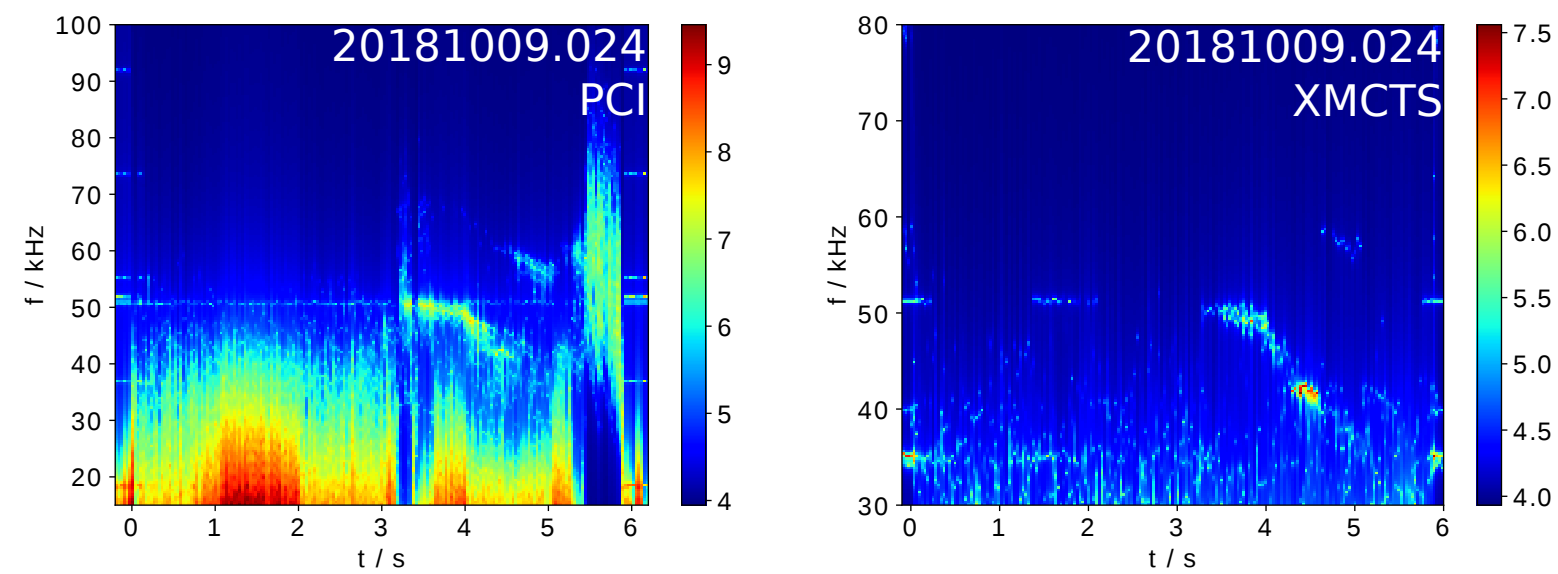

Figure 3: PCI (left) and XMCTS (right) see the same mode between 40 and $50 \mathrm{kHz}$ after the NBI tripped. With XMCTS it is also possible to determine the inversion radius. This suggests that the mode is core-localized.

was created by ECRH only. After this initial phase, ECRH was switched off and the plasma could be sustained for the following approximately five seconds with an injected NBI power of $P \cong 1.75 \mathrm{MW}$. The discharge was terminated at around $t \cong 5.9 \mathrm{~s}$ due to low diamagnetic energy. Since this operation phase was the first in which NBI became available, only one of the two beam boxes was in operation. Each NBI box contains 4 sources, but only 2 of them were active during this discharge, where they fired alternately for $1 \mathrm{~s}$ each. At around $3 \mathrm{~s}$, during the switching of the NBI sources, they tripped as can clearly be seen in the drop of diamagnetic energy (see grey shaded region in Fig. 2). These changes of the NBI parameters seemed to induce changes in the plasma (including the fast-ion distribution function) and Alfvén eigenmodes were excited in the plasma for $t>3 \mathrm{~s}$. Since these modes persisted for some time we choose the time point $t=3.9 \mathrm{~s}$ (when the mode activity is strong and the diamagnetic energy has almost made a recovery) for the analysis presented in this paper. This time point is indicated by the black vertical line in Fig. 2.

After $t=3 \mathrm{~s}$ a mode between 40 and $50 \mathrm{kHz}$ was seen in the PCI diagnostics. The same mode was also observed by XMCTS as can be seen in Fig. 3 on the left and right-hand side, respectively. While the PCI system operates with a limited number of sight lines (all with a very similar viewing angle), XMCTS has sight lines that cover the entire cross section of the plasma. An investigation determining the inversion radius suggests that this mode is very likely core-localized. Further evidence that the mode is localized in the core is given by the observed decrease of the mode frequency. During the discharge a rise of the core density (due to NBI fuelling) has been observed that is consistent with the down-sweeping of the frequency.

Different modes were observed by the magnetic diagnostics, in particular the Mirnov coils. Fig. 4 shows a spectrogram from a coil with very good signal to noise ratio that shows the modes very clearly. This particular pick-up coil is located on the outboard side of the machine. Coils in other poloidal positions on the same ring (same toroidal position) see the modes less clearly. As can be seen in Fig. 4, this Mirnov coil observes modes at $f \approx 190 \mathrm{kHz}, f \approx 150 \mathrm{kHz}$, and $f \approx 20 \mathrm{kHz}$ (visible after $t=3.0 \mathrm{~s}$ ). While the existence of so many modes at the same time is an interesting feature of this discharge that deserves to be mentioned, we will focus on the analysis of the $f \approx 190 \mathrm{kHz}$ mode as this mode is most readily assigned to a gap in the shear Alfvén spectrum. It is interesting that the PCI and the XMCTS diagnostics on the one hand and the Mirnov coils on the other hand observed different modes. Since they clearly vary in frequency it is possible that they are associated with different gaps in the shear Alfvén spectrum and are therefore also localized at different radial positions. The hypothesis, which will be proved later 


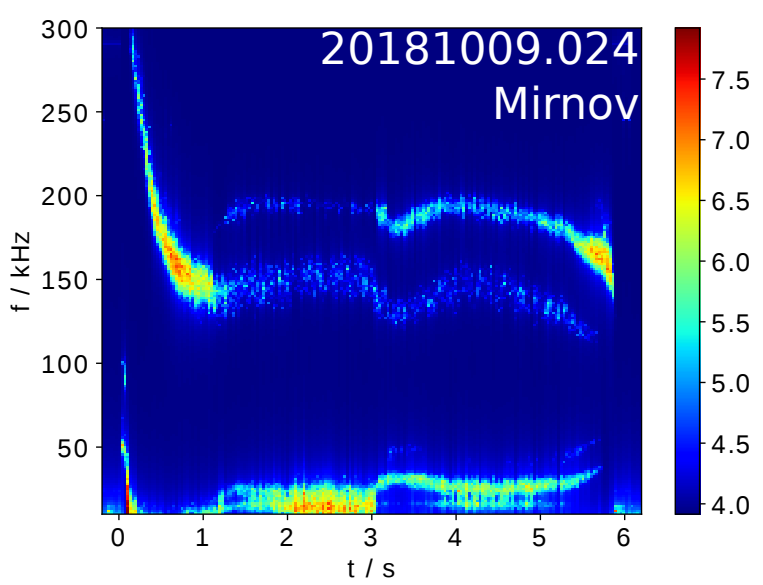

Figure 4: DMusic spectrogram of the signal recorded by Mirnov coil QXM11CE500x. This coil observes lowfrequency modes as well as MHD activity around 150 and $190 \mathrm{kHz}$. This diagnostics observes different modes than PCI and XMCTS do.
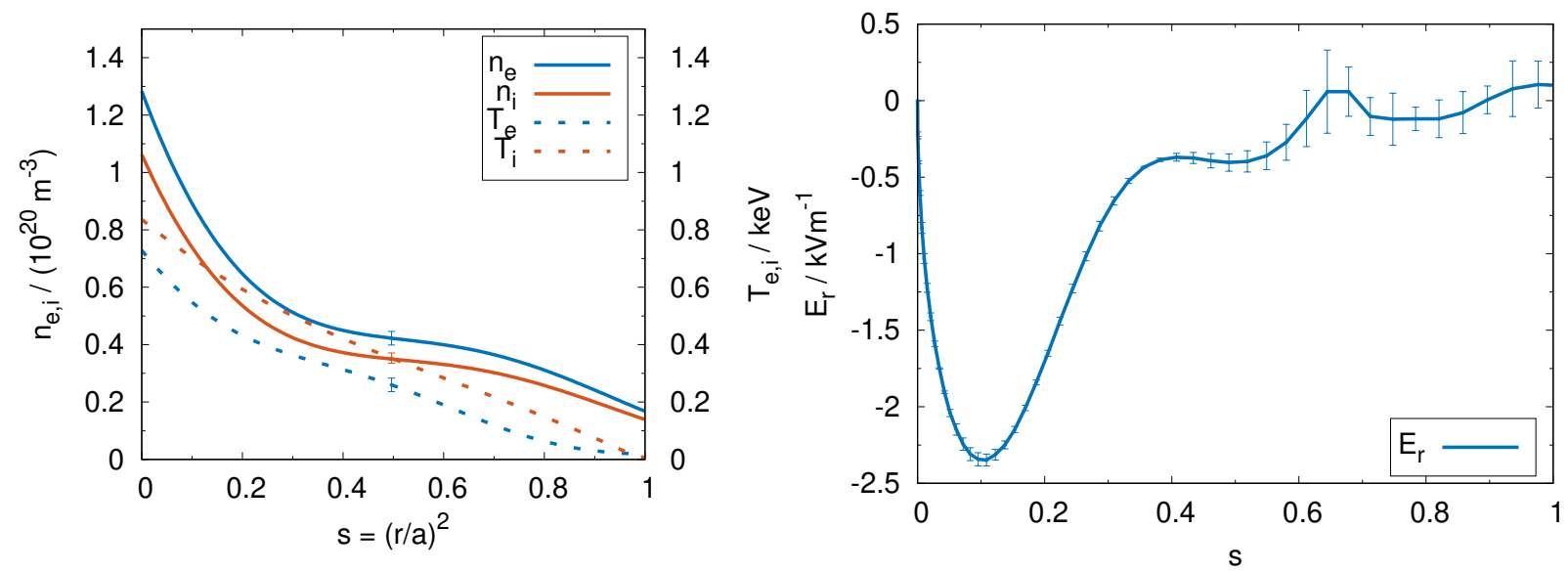

Figure 5: Left: Electron and ion densities and temperatures for experimental programme 20181009.024 at $t=3.9 \mathrm{~s}$. Right: Radial electric field determined by NTSS [31] calculations taking the profiles on the left-hand side as input. The plasma is in ion-root conditions throughout the radial domain.

in this paper, is that the Mirnov coils observe an edge-localized mode (the mode at $f \approx 190 \mathrm{kHz}$ ) with a frequency in the EAE gap while the XMCTS and PCI diagnostics detect a core-localized global Alfvén eigenmode (GAE) located below the TAE gap.

Even though the modes were first excited at $t=3.0 \mathrm{~s}$, they persisted until the end of the discharge. As stated before, we have chosen to perform the mode analysis at $t=3.9 \mathrm{~s}$ because the mode frequencies (as seen in the spectrograms) are roughly constant at this time and the overall level of mode activity is strong. Further, the diamagnetic energy has recovered again.

In order to compute the shear Alfvén continua, and to test if our hypothesis that the Mirnov coils and PIC/XMCTS observe modes in different gaps of the spectrum holds, we have to know the plasma profiles of experimental programme 20181009.024 at $t=3.9 \mathrm{~s}$. They are shown in Fig. 5. The profiles are generated from Thomson scattering $\left(n_{\mathrm{e}}\right.$ and $\left.T_{\mathrm{e}}\right)$ and chargeexchange recombination spectroscopy data $\left(T_{\mathrm{i}}\right) . n_{\mathrm{i}}$ is estimated using the measured $Z_{\text {eff }}$ value and assuming carbon as single impurity species. All profiles are mapped to the radial coordinate $s \equiv r_{\text {eff }}^{2} / a^{2}$ (normalized toroidal flux) using the best-fitting VMEC [32] equilibrium for this discharge from a data base of pre-calculated equilibria (In our case this is the equilibrium with 
the designation w7x_ref_337.). (Here, $r_{\text {eff }}$ is the effective radius of a flux surface and $a$ denotes the radius of the last closed magnetic surface.) However, this equilibrium does not yet take into account the experimentally measured shape of the pressure profile. A second self-consistent equilibrium, fully taking into account the kinetic pressure, is used in the remainder of this paper to compute the Alfvén continua, eigenmodes, and fast-ion distribution function. It can be understood as an improvement upon the initial equilibrium used for mapping the profiles. Characteristically for W7-X the iota profile of this equilibrium is close to unity with very little shear. Iota increases monotonically from $\iota \cong 0.87$ in the core to $\iota \cong 0.97$ at the edge.

The dashed lines on the left-hand side of Fig. 5 show the temperatures of the backgroundplasma ions and electrons. Compared to the average W7-X experimental programme from the last operation phase the temperatures are quite low. This is not surprising as the injected power was only $P \cong 1.75 \mathrm{MW}$ into a plasma with a volume of $V \cong 27 \mathrm{~m}^{-3}$. The density profiles of the two main species are shown as the solid lines. Here we see a strong peaking of the density on-axis. While most of the density profiles observed in W7-X in OP $1.2 \mathrm{~b}$ are very flat (ECRheated plasmas), this strong peaking seems to be a consequence of the fuelling provided by the NBI system. These profile effects of the NBI system are not the topic of this article and will not be further discussed here. We use the neoclassical transport code NTSS [31], which takes the plasma profiles as input, to calculate the neoclassical radial electric field, which is shown on the right-hand side of Fig. 5. This field leads to a poloidal $\mathbf{E} \times \mathbf{B}$-rotation of the plasma and thus causes a Doppler shift of the shear-Alfvén wave continuum. How this affects the structure of the continuum gaps and the frequencies of the eigenmodes within these gaps will be discussed in section 4.2 .

In order to obtain poloidal mode numbers of the experimentally observed modes, we use the SSI method to analyse the data obtained by 41 coils on a poloidally closed loop in section 11 of the machine. Thus, we hope to obtain a poloidal mode number associated with the mode at frequency $f \approx 190 \mathrm{kHz}$ prominently visible in the DMusic spectrogram, see Fig. 4 . The outcome of this analysis is shown in Fig. 6. Note, however, that the amplitude and phase calibration of the Mirnov probes is still ongoing which means that the poloidal mode numbers that are obtained by the SSI method have to be understood as first estimates. The left-hand side of this figure shows the frequencies that are common to all the different time traces recorded by the 41 Mirnov coils. By looking at the red circles, we can again identify the modes at $f \approx 150 \mathrm{kHz}$ and $f \approx 190 \mathrm{kHz}$ that we already observed in the DMusic spectrogram. The existence of these modes is robust, since they are still found when we increase the model order (red circles). In contrast, the modes close to zero frequency are not robust. Furthermore, the SSI method also gives us the mode structure in the space of the poloidally separated Mirnov probes. This is shown in the top-right plot of Fig. 6 , where the $x$-axis is the normalized poloidal angle and the $y$-axis is the signal amplitude in arbitrary units. The mode is localized on the outboard ('low-field') side of the device. Performing a Fourier transform of the signal amplitude (plot on the bottom-right), we can extract the dominant poloidal mode numbers. A rather broad maximum indicates mode numbers in the range $m \in[10,15]$, which we will use as a guidance in the search for Alfvén eigenmodes.

\section{Shear Alfvén continua}

For the computation of the shear Alfvén wave continuum we need high-quality data regarding the profiles and the magnetic equilibrium. As already mentioned in the previous section, we use the best fitting pre-calculated VMEC equilibrium for the mapping of profile data. This equilibrium is then used as a starting point for a second VMEC run in which the previously mapped pressure profile (see Fig. 5 for density and temperature) is fed back self-consistently into VMEC. We thus arrive at an equilibrium that is self-consistent with the experimentally measured profiles shown in Fig. 5. The fast-ion pressure is excluded since it is small compared 

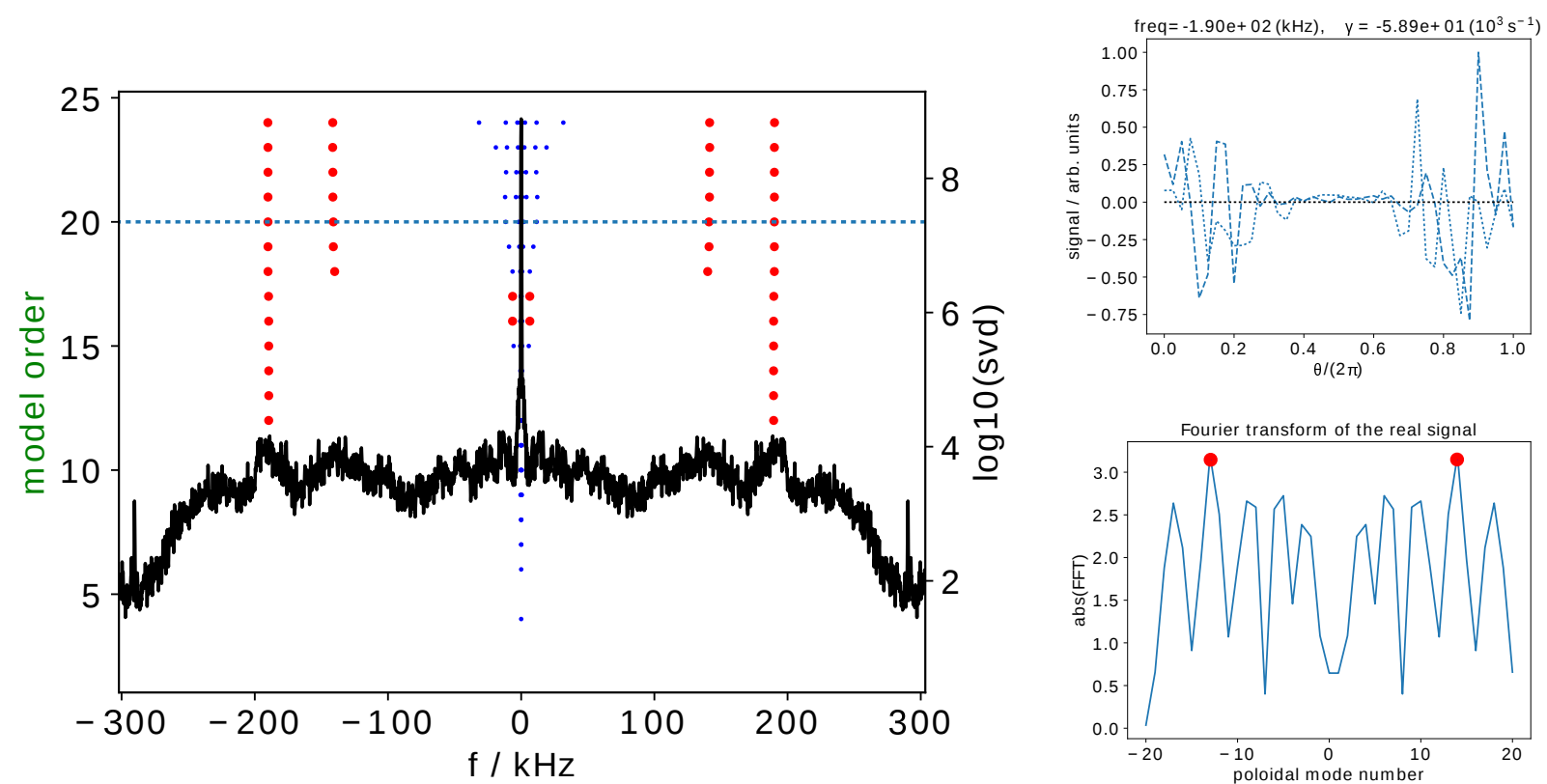

Figure 6: The SSI method to determine the poloidal mode number and the frequency of the mode observed in Fig. 4. Data from the time interval 4.0-4.5 s is used in this analysis. Left: Eigenmodes found by the SSI method (red circles) compared to the frequencies found by a singular value decomposition (black curve). The SSI method finds the mode at $f=190 \mathrm{kHz}$ and a second one (that can also be seen in Fig. 4 ) at $f \approx 150 \mathrm{kHz}$. Top-right: Mode structure in the frame of the poloidal array of Mirnov coils. The $x$-axis represents the normalized poloidal angle. Bottom-right: Fourier transform of the top-right plot (real component). The most likely poloidal mode number is $m=14$ (marked by a red circle).

with the pressure of the bulk plasma. Specifically, in the core of the plasma, the bulk-plasma beta is $\beta_{\text {bulk }} \approx 1.2 \%$, while the fast-ion beta is only $\beta_{\text {fast }} \approx 0.01 \%$.

We then use the CONTI code [25] to compute the shear Alfvén continua using a slowsound approximation [33] to include the effect of finite plasma pressure in a first approximation. Generally, when calculating the continuum, one needs to solve a linear system of equations for the radial component of the displacement vector and its divergence (see e.g. Ref. [25]). The coefficients of this linear system include terms related to Alfvén waves as well as sound waves. Both types of waves are coupled together via the geodesic curvature on the flux surface [33]. The so-called slow-sound approximation assumes that $\gamma p \ll \omega^{2} \rho$ (where $\gamma$ is the adiabatic index, $p$ is the pressure, and $\rho$ denotes the mass density) [33]. Using this approximation, the sound waves can be eliminated from the calculation (greatly reduces the numerical effort) while still retaining a low-order approximation of their effects on the shift of the Alfvén frequency gaps [33]. No such approximation is assumed for the (numerically much more demanding case) where the full coupling to sound waves is taken into account. This approach is more accurate for low frequencies and also permits the opening of additional gaps. Using the slow-sound approximation is valid if the Alfvénic frequencies are much larger than those of the sound modes. Although it is maybe not sufficient for the TAE gap in the core of the plasma, it should be much better for the EAE gap. Changes that come from a full coupling to sound waves as well as from the presence of the radial electric field (Doppler shift) will be discussed separately. Fig. 7 shows the results without Doppler shift and using the slow-sound approximation for the three distinct mode families of W7-X [34]. These mode families arise due to the three-dimensional shape of the plasma, which only enables modes with specific toroidal mode numbers to interact with each other. In W7-X these three mode families are labelled $N=0,1,2$. As an example, in the $N=0$ mode family only modes with toroidal mode numbers $n=\ldots,-10,-5,0,5,10, \ldots$ can couple. Couplings to 

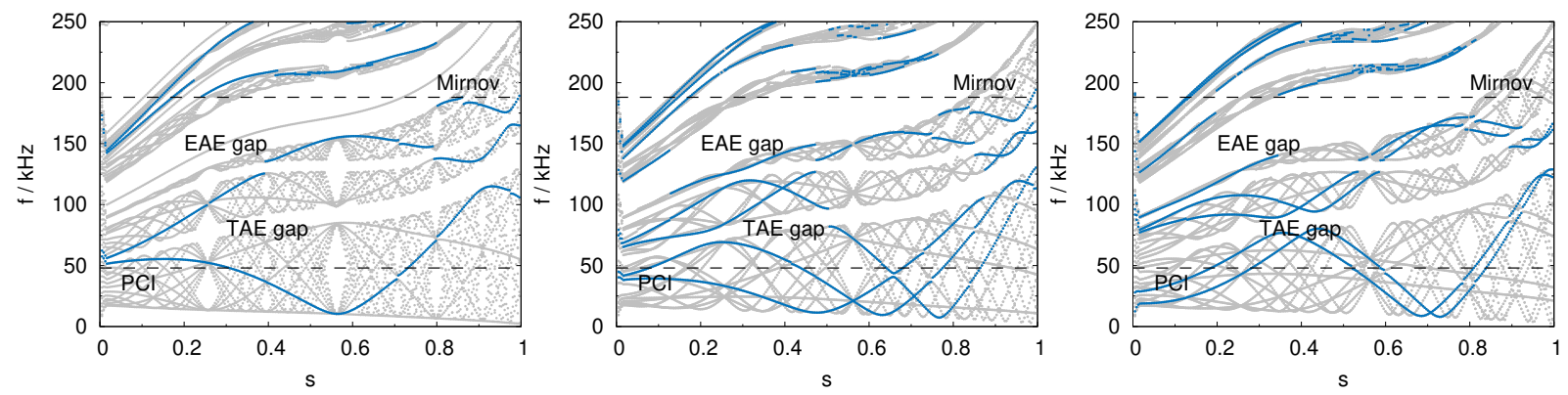

Figure 7: Shear Alfvén wave continua calculated by CONTI [25]. Continuum branches with poloidal mode numbers in the range $m \in[10,15]$ (corresponding to experimental measurements; see SSI method) are coloured blue. From left to right: $N=0, N=1$, and $N=2$ mode family. The gaps are at the same position regardless of the mode family, but the poloidal and toroidal mode numbers, which are coupled together, vary.

other values of $n$ are forbidden. For the $N=1$ and $N=2$ mode families, the couplings are different. More information on this topic is given in Ref. [34].

In Fig. 7 continuum branches with $m \in[10,15]$ have been coloured blue since the SSI method used to evaluate the Mirnov data suggested mode numbers in that interval. Two major gaps, the TAE and the EAE gap, are visible in the frequency range of interest. The frequencies of the modes observed by the Mirnov diagnostic fit into the EAE gap if we assume that the modes are localized in the outer portion of the plasma. This is a reasonable assumption since then the modes should be closer to the Mirnov coils, which should make them easier to observe. Note further that the SSI method suggests a mode located on the outboard side of the device. This is consistent with the observation that Mirnov coil QXM11CE500x (also located on the outboard side) shows a good signal-to-noise ratio. The mode that is seen by PCI and XMCTS is at much lower frequencies around $f=50 \mathrm{kHz}$. This puts the mode below the TAE gap throughout the entire radial domain. It thus seems unlikely that the mode is a regular TAE. Also an energetic particle mode (EPM), that is usually excited in the continuum and must overcome the continuum damping, can be ruled out due to the very low fast-ion pressure compared with the background-plasma pressure. We will find below that successfully assigning this mode to a gap in the spectrum will require that the full coupling to sound waves is taken into account.

Clearly, the continuum is a very useful (and computationally inexpensive) tool for the interpretation of experimental data and for assigning observed frequencies to specific gaps in the spectrum.

The continua have been calculated using only the $n_{\text {i }}$ profile as a measure of the mass density, neglecting any impurities for simplicity. The measured value for the effective charge of the plasma is $Z_{\text {eff }}=1.86$ which would globally reduce the frequencies of all Alfvén waves by $16 \%$, if we assume a carbon impurity. While this impurity source is certainly at the edge of the plasma, note that there is no profile information available regarding $Z_{\text {eff }}$. Thus, at present, we cannot discuss how the Alfvénic frequencies would shift locally in the plasma. This more detailed analysis would require an upgrade of the diagnostic systems at W7-X to more precisely measure the radial profiles of the various impurity species.

\subsection{Full coupling of sound and Alfvén waves}

So far, the continuum was calculated in the slow-sound approximation, which simplifies the calculation but is only accurate for the higher frequencies around the EAE gap. To look more closely at the TAE gap and the frequencies below, we perform another calculation for the $N=0$ mode family taking the full coupling of Alfvén waves and sound waves into account. The results are shown in Fig. 8. Note that even though the sound modes were present in the calculation, 

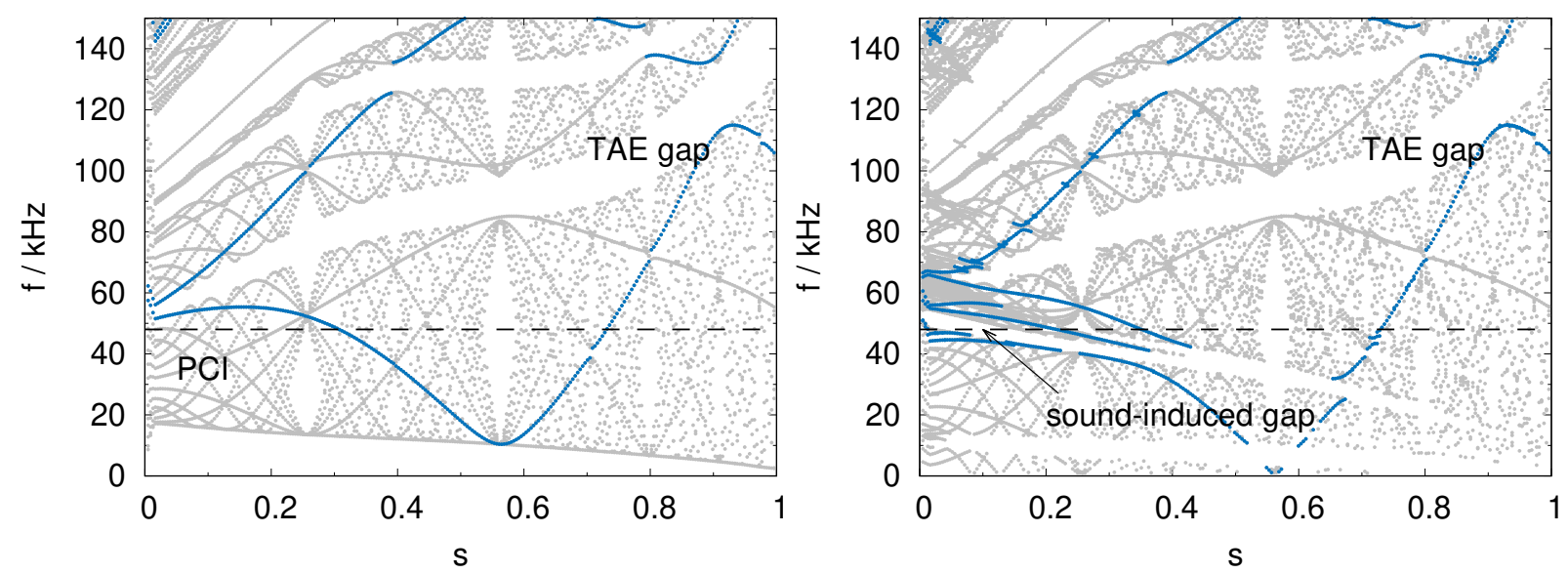

Figure 8: The effect of the full coupling to sound waves on the shear Alfvén wave continuum for the $N=0$ mode family. The left-hand side shows a zoomed-in view of the left-most plot of Fig. 7. The right-hand side shows, for the same case, the changes that come from the coupling to sound waves. (While the sound waves are present while solving the eigenvalue problem, they are not plotted here.) Most importantly, a new gap opens in the frequency range that matches the observations by PCI and XMCTS.

they are not plotted in Fig. 8 since the focus of this paper is on the Alfvén waves and not on the sound waves. By comparing the left-hand side of Fig. 8 (slow-sound approximation) to the right-hand side (full coupling to sound waves), it can be confirmed that especially in the core of the plasma, the presence of the sound waves leads to a slightly different shape of the TAE gap. Furthermore (and possibly more importantly) another gap is induced at a frequency of around $f=50 \mathrm{kHz}$ in the core of the plasma. The frequency and location of this sound-induced gap match the measurements by PCI and XMCTS very well.

Note further how the blue continuum branch right below this new gap has a maximum at $s \approx 0.1$ and $f \approx 50 \mathrm{kHz}$. This allows for the presence of a GAE mode slightly above this continuum accumulation point, which is also found in simulations with the CKA code (see below). Since the mode is located in a gap that is opened via the interaction with sound waves (i.e. finite plasma pressure) it could be considered a beta-induced Alfvén eigenmode (BAE). We, however, prefer the identification as a GAE (for the reasons stated above) that has minimal continuum damping due to the opening of a gap because of finite beta.

It should be mentioned that for this discharge the plasma pressure is low. The volumeaveraged beta (kinetic pressure divided by magnetic pressure) is only $\langle\beta\rangle=0.3 \%$. Thus, pressure effects are not very pronounced. This may change in later operation phases of W7-X that target plasmas with $\langle\beta\rangle=5 \%$.

Analogous changes of the continuum due to the interaction with sound modes are, of course, also seen for the other mode families in W7-X.

\subsection{The effect of a radial electric field}

Stellarators, like W7-X, exhibit a radial electric field in order to ensure ambipolarity of the neoclassical particle fluxes. This field can be calculated by neoclassical transport theory even if most of the transport is caused by gyrokinetic turbulence, because the latter is automatically ambipolar $[35,36]$. Depending on the conditions of the discharge this field can either be negative (ion root), positive (electron root) or both, with a zero crossing in between (like the core-electronroot-confinement, or CERC, discharges commonly observed in OP 1.1) [37]. This radial electric field leads to a poloidal $\mathbf{E} \times \mathbf{B}$ rotation of the plasma and therefore to a Doppler shift of the frequencies that are measured by the diagnostics in the laboratory frame. This naturally affects the radial shape of the shear Alfvén wave continua as it 'mixes' the various branches with 

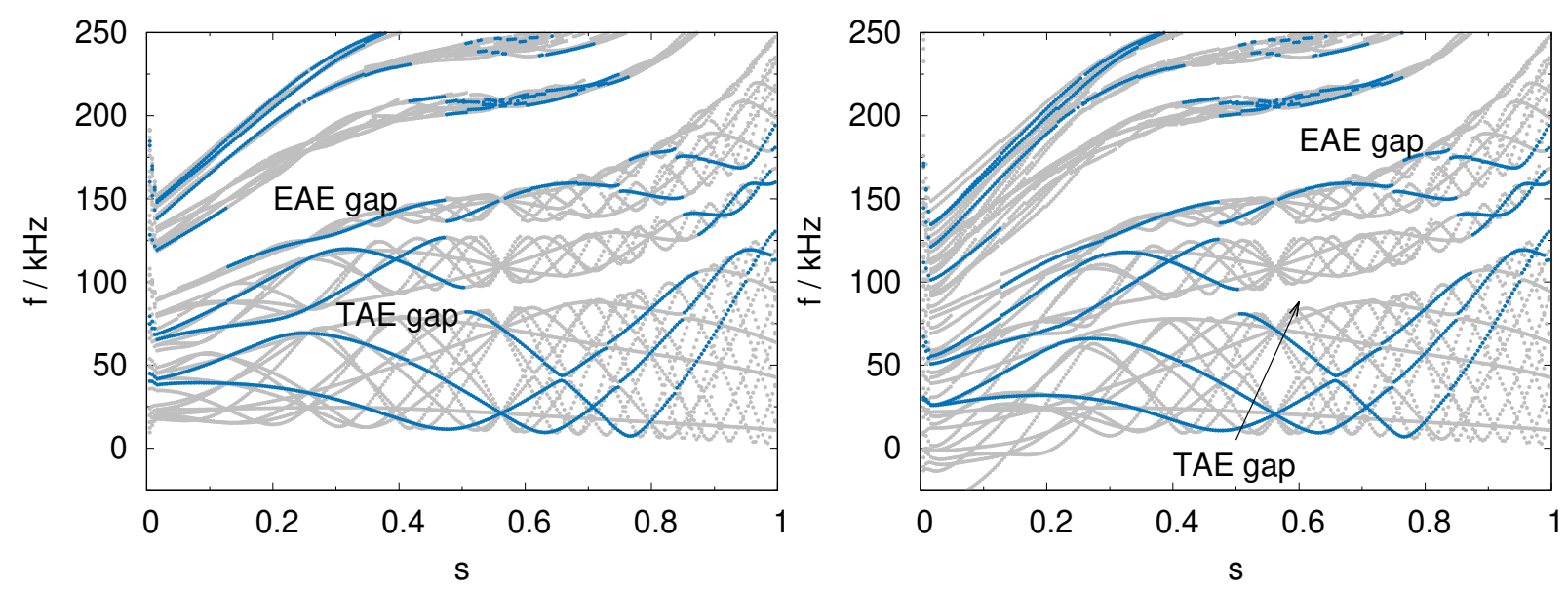

Figure 9: Influence of a radial electric field on the shear Alfvén continuum for the $N=1$ mode family. The left-hand side shows the same continuum as the middle plot of Fig. 7 (no Doppler shift) while on the right-hand side the radial electric field and the resulting Doppler shift are taken into account. While the total shift of the continuum branches depends on the mode numbers and on the radial location, it seems that the typical shift for this specific case is in the range of $\Delta f \approx-8.3 \mathrm{kHz}$. Even when a radial electric field is present, the gaps remain open (apart from the core) which is more clearly visible for the EAE gap.

different $m$ 's and can thus lead to frequency gaps potentially closing and opening locally.

The radial electric field in this discharge is calculated by the neoclassical transport code NTSS taking the plasma profiles shown in Fig. 5 as input. Knowing the radial electric field and the shear Alfvén wave continuum in the plasma frame (Fig. 7, $N=1$ mode family), we can transform this calculation to the laboratory frame by applying the Doppler shift (simple estimate)

$$
\omega_{\text {lab }}=\omega_{\text {plasma }}+\Delta \omega
$$

with

$$
\Delta \omega=v_{\mathbf{E} \times \mathbf{B}} k_{\perp} \approx \frac{E_{r}}{B} \frac{m}{a \sqrt{s}}
$$

to the previously calculated continuum. We thus arrive at an estimate for the Doppler shifted continuum shown in Fig. 9, where, for simplicity and to clearly show the effect of the Doppler shift, only a slow-sound approximation has been used in the continuum calculation. Looking at the right-hand side of the figure, it can be noted that the TAE gap closes in the core of the plasma, where $E_{r}$ is strong. On the other hand, the much wider EAE gap is affected less and remains open throughout the majority of the minor radius. Thus, apart from the core, the gaps remain open. Note that this conclusion is particular to this discharge with its radial electric field. Its radial variation is less than what is observed in other W7-X discharges thanks to the fact that this plasma is NBI-heated and is therefore in ion-root condition throughout the entire radial domain. If, on the other hand, we had a root transition (CERC discharges) the radial variation of $E_{r}$ and the resulting effect on the continuum would be much larger. Looking at the radial structure of $E_{r}$ in Fig. 5 one can observe a small hump at $s \approx 0.65$, which is probably unphysical. Nevertheless, the field is so small at this point that this feature does not impact the resulting Doppler shift significantly.

Even if the frequency gaps were to close due to the local Doppler shift, this might not necessarily mean that suddenly the modes acquire a large continuum damping rate: Consider the example of a low- $m$ mode number TAE. If the continuum gap is closed by a high- $m$ branch (that according to Eq. (6) is shifted much stronger), there will be no effect on the TAE since the mode numbers are widely different. This makes the interpretation of the Doppler-shifted 

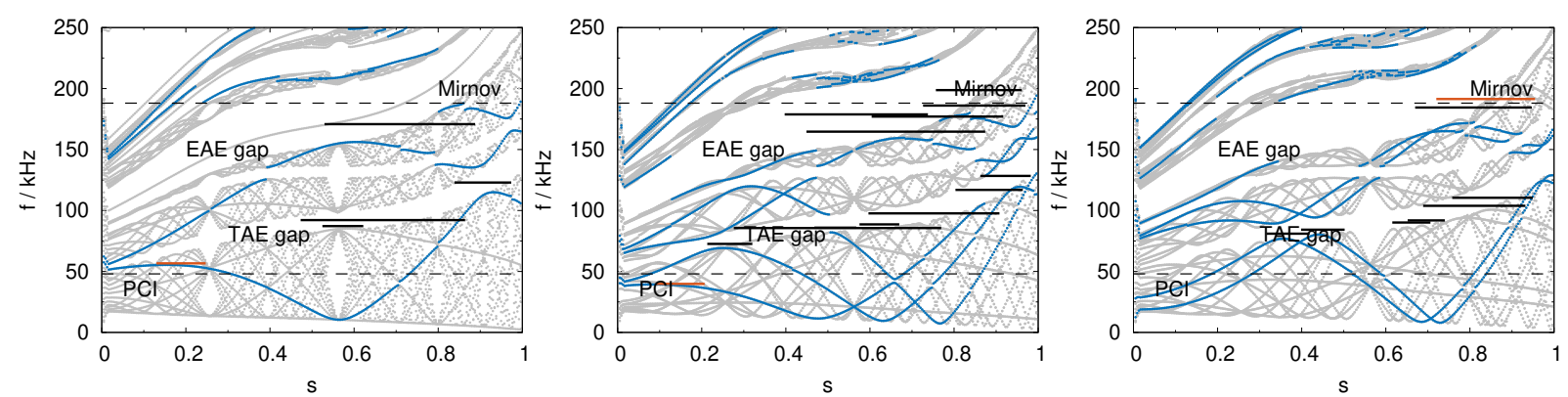

Figure 10: Same as Fig. 7, but also showing the modes that have been found by CKA. The length of each line indicates the FWHM of each mode. Note that due to the lack of experimental measurements of mode numbers and radial location, many of them have to be considered possible candidates to explain the measurements.
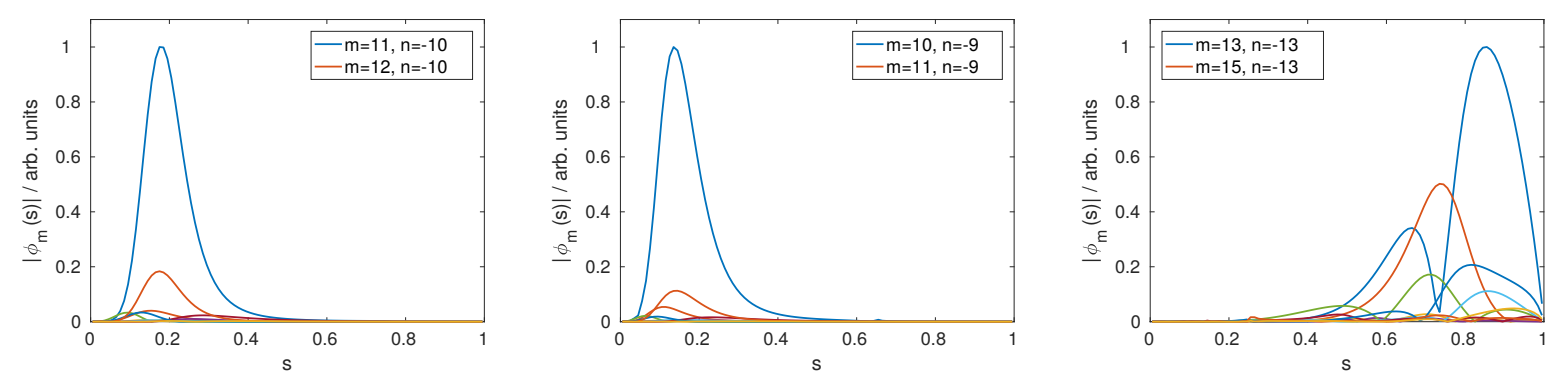

Figure 11: Radial mode structures of three of the modes found by CKA that best fit the experimental measurements in terms of their frequency. Left and middle plot: Two core-localized GAEs that have been found in the $N=0$ and $N=1$ mode families that could explain the measurements from PCI and XMCTS. Right plot: An edge-localized EAE that fits well to the mode observed by the Mirnov coils.

continua much more involved. Fully gyrokinetic simulations taking the radial electric field into account would be helpful to check if the damping rate of a given mode changes due to the presence of $E_{r}$.

\section{Alfvén eigenmodes in the frequency gaps}

We use the ideal-MHD code CKA to purposefully look for Alfvén eigenmodes in the frequency gaps of the previously calculated continuous spectra. In this process we are guided by the experiment in terms of the expected frequencies and (to a lesser extend) the poloidal mode numbers. Since no information is available for the toroidal mode numbers, we perform the search for each of the three mode families looking for eigenmodes close to the TAE and EAE gap that have poloidal mode numbers below $m=20$. Recall that the SSI method (roughly) suggested $m \in[10,15]$.

A total of 26 modes (spread over the three mode families) that fit our search criteria are found by CKA. Rising in frequency we find core-localized GAEs, TAEs, and EAEs. Experimental constraints for the mode numbers and the radial positions of the modes could strongly help to limit the modelling efforts to modes most likely seen in the experiment.

The frequencies of all the modes found by CKA as well as their radial positions (FWHM) are indicated in Fig. 10. Among these possible candidates that could explain the experimental measurements, we highlight three modes (one per mode family) that show very good agreement with the experimental measurements in terms of their frequency. Their radial mode structures are shown in Fig. 11. These are two core-localized GAE-type modes (a single poloidal Fourier harmonic dominates their radial mode structure, see Fig. 11) found in mode families $N=0$ and $N=1$ as well as an edge-localized EAE found in mode family $N=2$. Note that a small 

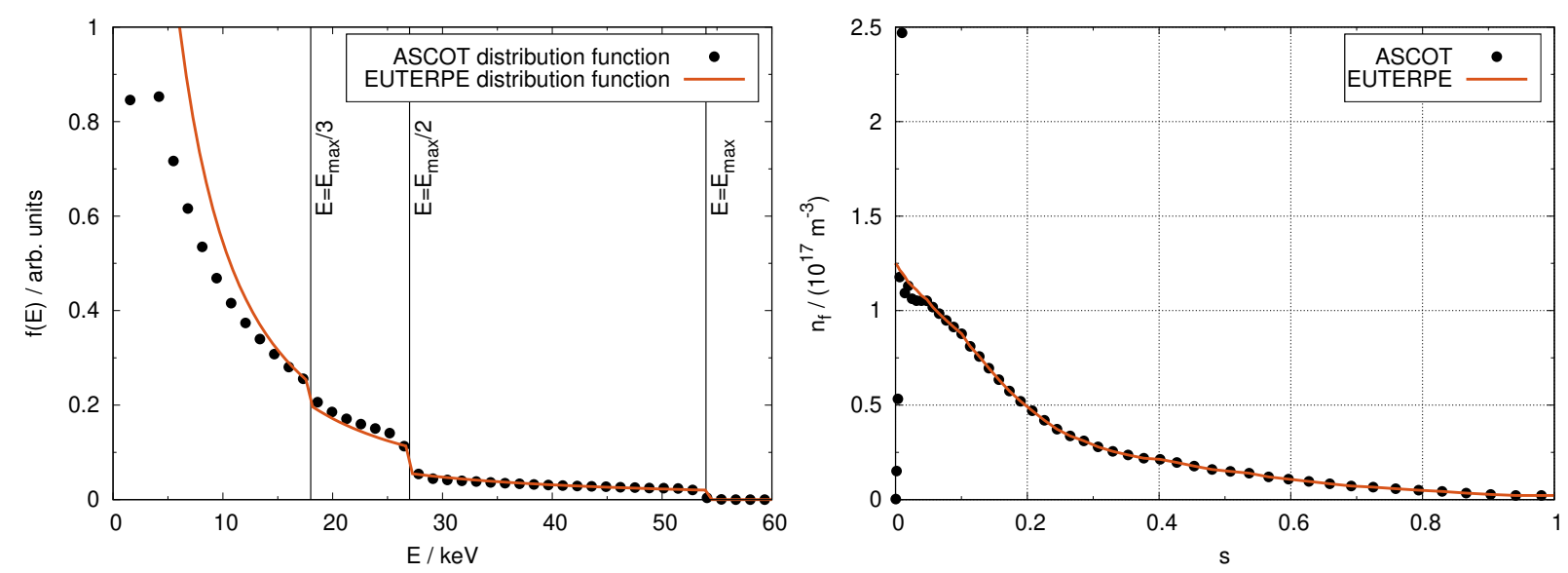

Figure 12: Fast-ion distribution function (left) and radial density profile (right) computed by ASCOT for the background-plasma profiles shown in Fig. 5. The black dots are the ASCOT simulation results, while the red lines indicate the fits used in the later CKA-EUTERPE simulations. Note that the fast-ion distribution function computed by ASCOT is anisotropic in the pitch-angle variable. To gain more flexibility, in EUTERPE we use an isotropic slowing down (shown here) as well as a model for an anisotropic slowing-down distribution function (shown later).

amount of side-band coupling is not unusual for GAEs. The GAEs match the observations from PCI and XMCTS while the EAE can explain the measurements by the Mirnov coils. These 'best-matching' modes are shown with red colour in Fig. 10.

In the CKA modelling we find a number of modes with frequencies in the TAE gap. Referring back to Figs. 3 and 4, we do not observe any mode activity in this frequency range. We may thus conclude that it seems unlikely that the modes observed in the experiment are TAEs.

\section{Fast-ion distribution function}

In order to calculate which of the many modes found by CKA can be destabilized by the fast ions (if any), we need to calculate their distribution function. This is done using the ASCOT code, which uses the total NBI heating power of this discharge as well as the profiles and the radial electric field specified in Fig. 5 as input. While ASCOT is capable of calculating many interesting effects related to the fast ions (e.g. fast-ion heat load on the wall), for our purposes we need a distribution function in velocity space as well as a radial fast-ion density profile. This information is displayed in Fig. 12 on the left and on the right, respectively. Note that ASCOT provides an anisotropic fast-ion distribution function in velocity space that ultimately has its cause in the NBI injection geometry at W7-X. However, to gain more flexibility in the EUTERPE simulations, we summed over all pitch angles and only consider an isotropic distribution function in energy space. For this type of function an analytical model is implemented in EUTERPE. We use the ASCOT simulation to determine the parameters of the model such that the EUTERPE distribution function fits the (isotropic) ASCOT simulation (see left-hand side of Fig. 12).

This approach of using ASCOT to determine the parameters of model distribution functions within EUTERPE allows for a higher degree of flexibility since this enables us to directly compare e.g. isotropic distribution functions like the one shown above with other distribution functions where we control the degree of anisotropy (see Fig. 15 below).

As already mentioned in the introduction, the two sources in beam box NI21 were switched every $1 \mathrm{~s}$. This is much longer than the slowing-down time, $\tau_{\mathrm{s}} \cong 6.15 \mathrm{~ms}$ (calculated at $s=0.25$ ), of the fast ions, which means that for the time point we analyse, $t=3.9 \mathrm{~s}$, we can assume the fast-ion distribution function to be fully developed.

Looking at the distribution function, it can be seen that, even though the maximum injection 


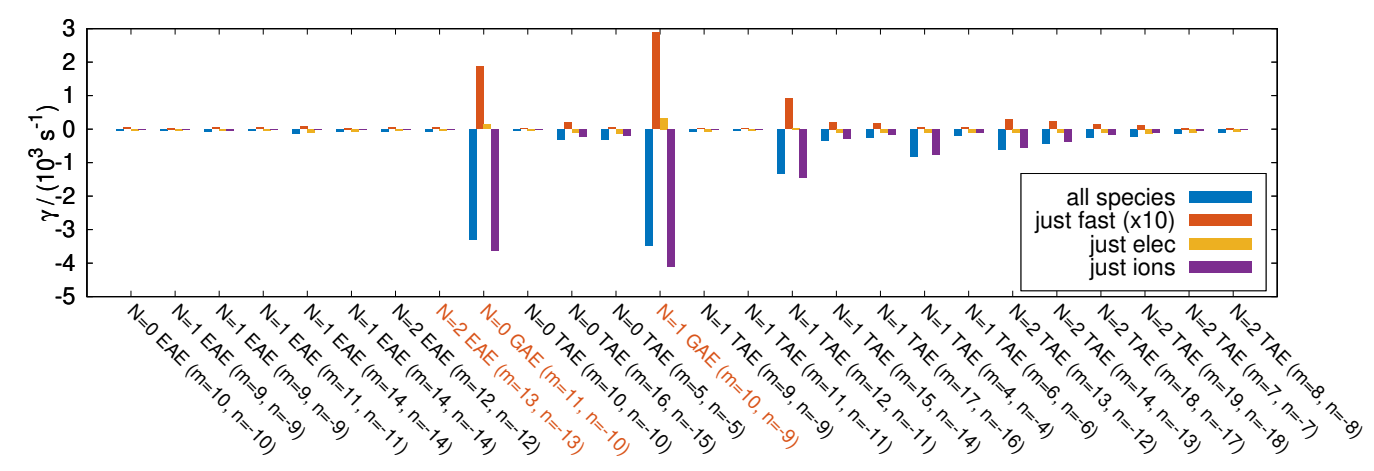

Figure 13: Growth/damping rates of all the modes found by CKA-EUTERPE. The modes that best fit the experimental measurements are labelled in red. Overall, CKA-EUTERPE predicts that all modes are stable. Regarding the fast-ion drive, two modes clearly react most strongly to the fast ions: The two core-localized GAEs that match the measurements from XMCTS and PCI.

energy of the NBI system is $55 \mathrm{keV}$ for protons, most of the particles have much smaller energies. This is primarily due to the low electron temperature, which leads to a low value for the critical velocity. At $s=0.25$ (mid-radius) the fast particles have an average temperature of only $9.7 \mathrm{keV}$ and the critical velocity is $v_{\mathrm{c}} \cong 9.97 \cdot 10^{5} \mathrm{~m} / \mathrm{s}$. The radial fast-ion density gradient, which is the source of free energy to destabilize fast-ion-driven modes, is largest in the core of the plasma. We therefore expect the most strongly driven modes in this region. Nevertheless, both the fast-ion energy as well as their density are relatively low (compared to typical conditions in similarly sized machines) which is partly due to the low NBI power used in this discharge. Thus, at mid-radius the fast-ion beta is $0.0023 \%$ while at the same position the background-plasma beta is about two orders of magnitude larger. Therefore, no strong fast-ion drive can be expected, even in the region of the strong fast-ion density gradient.

\section{Drive of the modes}

In order to compute the growth (or damping) rates of the modes due to the interaction with kinetic species in the plasma, we employ the perturbative CKA-EUTERPE model, which computes the power transfer of the kinetic particles to the mode with a fixed mode structure previously calculated by CKA. For a description of the model itself and its capabilities see Refs. $[2,26,28]$. We use the perturbative model here, because, compared to fully gyrokinetic simulations, it is numerically very stable and inexpensive, so that it can be used to assess the stability of the many modes that we found in Sec. 5. The results for each of the 26 modes found by CKA are depicted in Fig. 13. As this figure contains a lot of information, we have shown the modes that have previously been identified to best fit to the experimental measurements in red. Note further how the red bars in the figure, corresponding to the drive due to the fast ions, are enlarged by a factor of ten. This is necessary in order to make it more clearly visible because the fast-ion pressure in this discharge is so much lower than the pressure of the background plasma.

By examining Fig. 13 closely, we come to the conclusion that the CKA-EUTERPE modelling paints an ambiguous picture: We observe that, in general, effects of the background plasma dominate over that of the fast particles, which is understandable since the fast-ion beta is low. On the other hand, the modes shown in Fig. 3 are not present from the beginning of the discharge, but are excited only after the NBI sources trip. Thus, there are modes where the observations suggest that their existence is tied to fast-ion effects - a fact that the CKA-EUTERPE modelling cannot reproduce quantitatively by simply calculating the fast-ion drive. Almost none of the TAEs react strongly to the fast ions, which is consistent with experimental measurements. 
The background plasma ions and electrons were modelled with a Maxwellian distribution functions. Here, the modes are stabilized by the interaction with the background plasma. Note, however, that for a different set of plasma profiles (not shown here) the background-plasma electrons were able to destabilize a few of the TAEs. We will revisit this issue in a future publication.

Nevertheless, while we cannot draw any clear conclusion from the CKA-EUTERPE modelling about why certain modes are observed in the experiment while others are not, it is not a completely futile exercise. Rather, the CKA-EUTERPE modelling is the most sensitive step of this whole analysis. Keep in mind that the main purpose of this article is to show an 'integral view' of our current modelling capabilities. This includes data analysis, calculating the continua, assigning the observed modes to gaps in the spectrum, and assessing the stability of these modes. Thus, in order to find out which parts of this analysis chain work well and which part needs more work, it is important to use data that resemble the actual experiment as closely as possible. Because the CKA-EUTERPE modelling is the most sensitive step in this whole analysis, it reacts most strongly to slight inaccuracies in the equilibrium (including the iota profile), the profiles, and, of course, the fast-ion distribution function. In this way the CKA-EUTERPE modelling done here emphasizes that we need high-quality data in order for such simulations to be successful.

Even when parts of this whole analysis chain (such as the CKA-EUTERPE modelling done here) turn out to be in partial disagreement with the experimental measurements, it is still important that such tests are done and properly communicated.

Thus, while there are discrepancies between the CKA-EUTERPE modelling and the experiments that clearly show that more work will be required in the future, there are other aspects of the modelling that work quite well to date. We observe, for instance, that as far as the fast-ion drive is concerned there clearly are two outliers from the general trend of very low growth rates. We observe that the two core-localised GAEs, whose frequencies match the experimental observations by PCI and XMCTS (and for XMCTS also the radial position), react most strongly to the presence of the fast ions. Thus, while we are unable to quantitatively predict their exact growth and damping rates at this stage, by just looking at the fast ions, the simulations may explain why these modes in particular were excited after the NBI sources tripped. The fundamental reason why these modes are most strongly driven by the fast ions in the CKA-EUTERPE modelling is the shape of the fast-ion density profile in combination with the mode location: Both modes are localized in the core of the plasma where the fast-ion density gradient is strongest.

Note that no toroidal mode numbers are available from the experiment. Therefore we cannot decide which of the two candidate-GAEs was actually observed in the discharge. Further, note that we cannot precisely model what happened to the NBI system at around $t=3.0 \mathrm{~s}$, which leads to an obvious uncertainty in the fast-ion distribution function.

The situation is slightly different for the edge-localized EAE that is observed by the Mirnov coils. The simulations reveal that it is only very slightly driven by the fast ions. At the same time, its damping due to the background plasma is also small while still outweighing the fast-ion drive. Since the fast-ion density gradient is low in the region where this mode is localized, it seems in principle possible that this mode is a good candidate to be driven by background-plasma electrons or ions, although the current simulations with the present profiles do not support this conclusion. This could, again, point to inaccuracies in the plasma profiles or, because the mode is so edge-localized, inaccuracies of the magnetic equilibrium at the edge (e.g. caused by the presence of islands). 

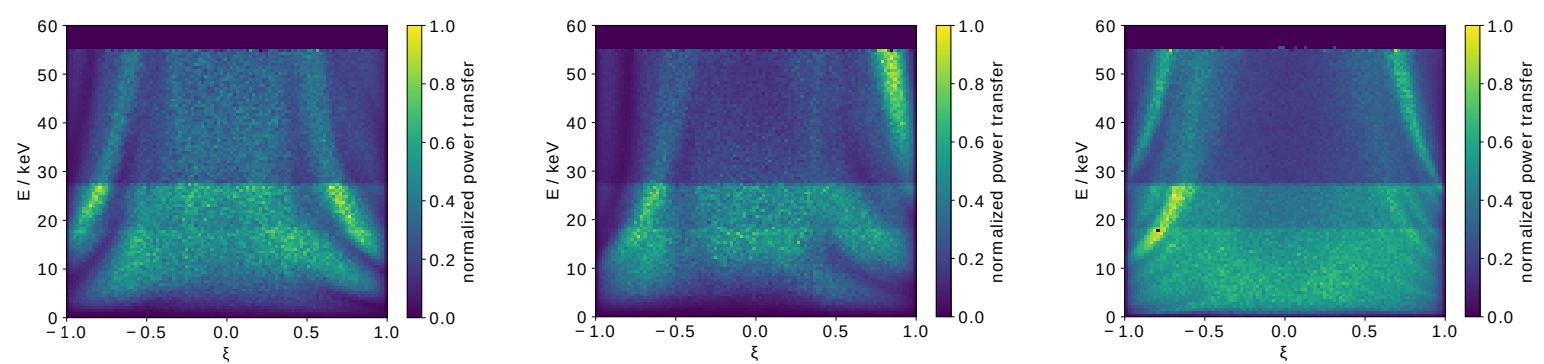

Figure 14: Normalized absolute value of the power transfer from the fast particles to the AEs in velocity space $\left(E, \xi=v_{\|} / v\right)$. Left: $\operatorname{GAE}(N=0)$, Middle: GAE $(N=1)$, Right: EAE $(N=2)$. The resonances are different for each mode but definitely favour strongly passing fast particles.

\subsection{Power transfer in velocity space}

It is also possible to investigate the power transfer between the particles and the modes in velocity space. In order to more clearly draw conclusions regarding the NBI system of W7-X, we limit ourselves here to the fast ions only. The results are shown in Fig. 14. We observe that the resonances are in different positions for each of the modes. The core-localized GAE from the $N=0$ mode family is driven by medium-energy co- and counter-passing particles. The equivalent mode from the $N=1$ mode family has a strong resonance for high-energy co-passing fast ions. The edge-localized EAE shows multiple resonances in the high-energy range.

All modes have one similarity: They are predominantly driven by fast ions with pitch angles $|\xi| \gtrsim 0.6$, which implies passing orbits. Hence, an experimental scenario that exhibits a strong drive of these modes would require passing fast ions. Looking at W7-X, these are just not the typical operating conditions. In the upcoming operation phase OP 2, which is supposed to start at the end of 2020, the second beam box will be operational. The typical fast-ion distribution function that is expected in these scenarios peaks around a pitch angle of $\xi_{0}= \pm 0.4$ as a direct consequence of the injection geometry. We can thus conclude that a strong drive of these modes, which seem to favour passing particles, is unlikely.

Nevertheless, an anisotropic distribution function introduces additional gradients in velocity space that may potentially change the drive of the modes. Here we can try to model what the resulting effects could be.

\subsection{Effects of an anisotropic distribution function}

Thus far, we have used an isotropic distribution function for the fast ions. However, as stated in the previous section, the injection geometry at W7-X leads to an anisotropic distribution function which typically peaks around $\xi_{0}= \pm 0.4$. In order to model the effects of velocity-space gradients on the growth of the modes, we use a model distribution function of the form

$$
f_{0}=C \sum_{i=1}^{3} \frac{w_{i}}{v^{3}+v_{\mathrm{c}}^{3}} H\left(v_{b, i}-v\right) \sum_{j= \pm 1} \exp \left[-\frac{\left(\xi-j \xi_{0}\right)^{2}}{2 \sigma^{2}}\right]
$$

where $\xi_{0}$ and $\sigma=0.2$ describe the region where this distribution function peaks in terms of $\xi=v_{\|} / v$ and the degree of anisotropy, respectively. The $w_{i}$ are weights, $H$ denotes the Heaviside step function, $v_{b, i}$ are the beam velocities, $C$ is a normalization constant, and $v_{\mathrm{c}}$ is the critical velocity defined as in [38]. Setting the second factor, the sum over $j= \pm 1$, to unity we retain the previous isotropic slowing-down distribution function.

How the isotropic distribution function, as well as two anisotropic ones with $\xi_{0}=0.5$ and $\xi_{0}=0.9$, respectively, look like in velocity space is shown in Fig. 15. If we make the distribution 

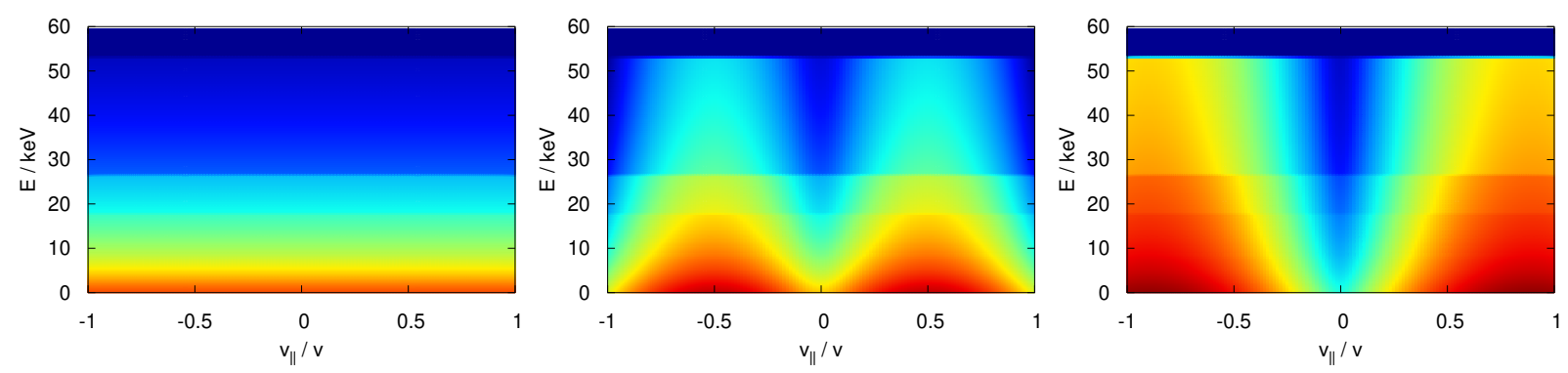

Figure 15: Isotropic and anisotropic distribution functions for the fast ions. By making the distribution function progressively more anisotropic we generate more passing particles thus increasing the drive of the modes.

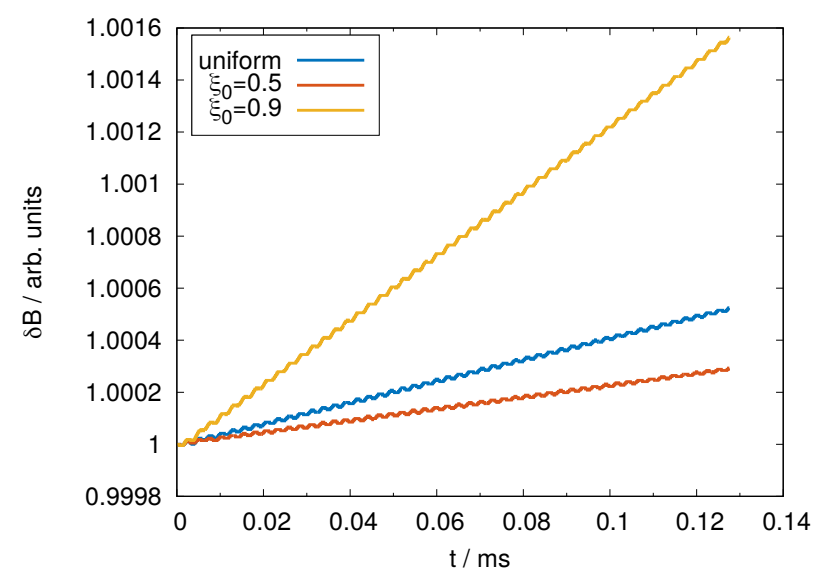

Figure 16: Increase of the linear growth rate of the edge-localized EAE with increasing degree of anisotropy of the fast-particle distribution function. A very anisotropic distribution function with much more passing particles can increase the growth rate of the mode by roughly a factor of three.

function more and more anisotropic, we push more particles into those regions of velocity space where resonances of the modes with the particles lie. Doing so should increase the fast-ion drive. Note, however, that this is particular to the modes studied in this paper with the resonances as shown in Fig. 14. The middle plot of Fig. 15 is a good approximation of what can be expected for the upcoming operation phase of W7-X when the second beam box becomes available.

In order to prove that more passing particles would be advantageous for exciting AEs, we can perform a set of simulations for a given mode with different distribution functions. For this purpose, we choose the edge-localized EAE mode, which very clearly interacts with strongly passing fast ions. The results are shown in Fig. 16. The figure confirms that strongly passing particles would be needed to really drive the modes unstable. For this particular mode, the growth rate increases by roughly a factor of three. On the other hand, a smaller degree of anisotropy (as it can be expected for future operation phases of W7-X) actually reduces the growth rate of this mode. (see red curve corresponding to $\xi_{0}=0.5$ ). (We observe, however, that this is not the case for the two core-localized GAEs.) Thus, this investigation shows the sensitivity of the results to the parameters of the distribution function, indicating that it must be known to a high degree of accuracy if reliable estimates for growth and damping rates are to be provided by gyrokinetic codes.

It should further be mentioned that, even when we increase the degree of the anisotropy of the distribution function as shown here, $f_{0}$ is still symmetric in $\xi$ which means that there is no equilibrium current (due to the fast ions, but also in general) included in the modelling. We therefore explicitly exclude the possibility of current-driven modes, which are outside the 
scope of this paper. It has, however, been observed in W7-X that strong electron-cyclotron current drive (ECCD) can trigger modes that can even lead to abrupt termination events of the plasma [39]. Modelling results of those cases will be reported elsewhere. Note further that also unbalanced NBI can drive a current in the plasma that can distort the iota profile and affect the stability of AEs. It then becomes necessary to separate the effect of 'pure' neutral-beam current drive from changes in the bootstrap current caused by profile changes due to NBI heating and fuelling. Again, these effects are outside the scope of this paper.

\section{Summary and conclusions}

This paper addresses the first investigation of mode activity in the recent NBI operation of Wendelstein 7-X (W7-X). During the last operation phase (OP 1.2b conducted from July till October 2018) the NBI system was commissioned and generated fast ions in the device for the first time. MHD mode activity was observed in a discharge that featured longer NBI pulses, which were primarily designed to study NBI heating. However, mode activity was also observed in other discharges, in which the plasma was only heated by ECRH. Investigations on how the electron-pressure gradient can potentially destabilize Alfvén eigenmodes (AEs) in W7-X are still ongoing.

Here, we use the NBI-only discharge 20181009.024, which showed rich mode activity in different frequency ranges, for the first validation exercise of our tools for data analysis as well as for our modelling tools. Mode activity is observed by three different systems: The Mirnov coils observe activity around $f=190 \mathrm{kHz}$, while the PCI and XMCTS systems see a different mode between 40 and $50 \mathrm{kHz}$. To analyse the available data with the main objective to accurately determine the mode frequencies and spatial structures, we introduce two advanced algorithms DMusic and SSI - that are well known for signal-processing applications, to the realm of plasma physics.

The application of these novel tools enabled us to systematically extract the mode frequencies and poloidal mode numbers from the experimental data. These information are then used for guidance in the subsequent modelling.

The modelling efforts start with the application of the CONTI code to calculate the shear Alfvén wave continua. For a higher degree of accuracy, a magnetic equilibrium - calculated by the VMEC code - that is consistent with the measured pressure profile is used in the simulation. We emphasize again that such an approach of iterating upon the initial equilibrium is necessary for the highly sensitive MHD phenomena that are the focus of this paper.

We studied the effects that the full coupling of sound and Alfvén waves have on the shear Alfvén continuum. We find that taking this coupling into account leads to the formation of a sound-induced gap precisely at the correct position to explain the frequency measurements by PCI and XMCTS. Further, the presence of a neoclassical radial electric field is investigated. It leads to a Doppler shift of the continuum branches due to the poloidal $\mathbf{E} \times \mathbf{B}$-rotation of the plasma. We can confirm that the frequency gaps remain open and that the average frequency shift is only on the order of $\Delta f \approx-8.3 \mathrm{kHz}$ for our specific case.

The structure of the shear Alfvén continua is compatible with the initial hypothesis that the mode observed by the Mirnov coils is an edge-localized EAE while the modes seen by the PCI and XMCTS systems are core-localized GAEs. Therefore, we conclude that the continuum is a powerful and comparatively inexpensive tool for the initial analysis of experimental data.

Continuing the investigation in more detail, we find a great number of Alfvén eigenmodes in the gaps of the continuous spectra using the CKA code for the simulations. Given the limited amount of information from the experiment (e.g. lacking information about toroidal mode numbers), most of these modes have to be considered as possible candidates that could potentially explain the experimental measurements. Finding these modes is still a very hands-on process and more experimental guidance in terms of mode numbers and radial location of the 
observed modes would be helpful. We proceed to highlight a couple of modes that best fit to the measurements in terms of frequency and focus on them in the following analysis.

In order to investigate gyrokinetically if it is possible for the fast ions to destabilize these modes, a fast-ion distribution function is needed. We use the ASCOT code to calculate such a distribution function for the conditions of discharge 20181009.024 at the time point $t=3.9 \mathrm{~s}$. In this experimental programme, the fast-ion pressure is very low compared with the pressure of the background plasma - a fact which manifests itself also in the gyrokinetic simulations, performed with the perturbative model CKA-EUTERPE, with very low growth rates due to the fast ions. Overall, kinetic bulk-plasma effects are much stronger in the current discharge where they are found to act stabilizing. In particular, the fast-ion drive does not overcome this background-plasma damping.

Thus, while there are some problems in the quantitative calculation of the growth and damping rates of the modes, the qualitative pictures regarding the fast ions is quite clear: In the simulation we find two modes that react much more strongly than the others to the fast ions and these appear to be the core-localized GAEs that are observed experimentally. In this particular discharge the modelling suggests that the background plasma is unable to destabilize the observed modes. However, as we have seen that this conclusion depends sensitively on the exact profiles, we cannot rule out the possibility that the electron-pressure gradient may destabilize some modes in other discharges, especially ones without NBI heating. Note that mode activity has been observed in other experimental programmes that were heated exclusively by ECRH. This issue of modes driven by the background plasma will have to be addressed in more detail in the future.

The fact that the CKA-EUTERPE modelling does not predict any unstable modes may thus point to inaccuracies in the equilibrium, the profiles, or the fast-ion distribution function. The fact that unstable modes were observed experimentally clearly shows that more work is needed regarding this step of our analysis chain. Nevertheless, as this article reports on the first validation exercise for our entire tool chain (including CKA-EUTERPE), it is important to use data as realisitic as possible - precisely to find out which parts of the analysis work well and which need more work. We want to mention here that, as part of a sensitivity analysis, we increased the fast-ion density and found a proportional increase of the growth rates. But this introduces a degree of arbitrariness, because this fast-ion distribution function deviates from the one that, according to ASCOT, is consistent with the background plasma. Hence, we chose to use a consistent distribution function instead. We also investigated the effects of an anisotropic fastion distribution function on the growth rate of the edge-localized EAE. In part this sensitivity analysis is motivated by trying to find operation regimes with increased fast-ion-induced mode activity in future operation phases. Because the locations of the resonances with the fast ions in velocity space favour strongly passing particles, we can easily increase the growth rate of this mode by roughly a factor of three by making the distribution function progressively more anisotropic and pushing more particles close to the resonances. This indicates that accurate distribution functions are required for the kinetic modelling in order to predict the growth and damping rates. Obtaining such distribution functions experimentally could be a challenge in the future. Thus, at the moment the fast-ion distribution function is determined numerically by ASCOT. In the future, a validation exercise should be undertaken in order to confirm that the ASCOT distribution function agrees with experimental measurements of (moments of) the distribution function. This is especially important since we identified here that the fast-ion drive calculated by CKA-EUTERPE, using a distribution function calculated by ASCOT, is too low to explain the experimental measurements. The correctness of ASCOT for predicting fast-ioninduced heat loads on the first wall of $\mathrm{W} 7-\mathrm{X}$ has already been confirmed $[9,40,41]$. We saw that, for an anisotropic distribution function that is close to our expectation of typical conditions we will have in the next operation phase of W7-X (when the second beam box becomes operational), 
no strong increase of the growth rates is observed. This shows the general difficulty of exciting AEs by fast particles in W7-X.

In future work, the kinetic results for the growth and damping rates of the modes that are obtained here, should be checked with fully gyrokinetic simulations that could be performed with EUTERPE. However, such simulations might be numerically challenging due to the low fast-ion density and the resulting low growth rates which could be susceptible to numerical noise.

\section{Acknowledgement}

Simulations were carried out at the MARCONI supercomputer system of EUROfusion hosted at CINECA in Italy as well as on the local Linux cluster in Greifswald, where support of Henry Leyh is appreciated.

We would also like to thank the anonymous referees for their insightful comments.

This work has been carried out within the framework of the EUROfusion Consortium and has received funding from the Euratom research and training programme 2014-2018 and 2019-2020 under grant agreement No 633053. The views and opinions expressed herein do not necessarily reflect those of the European Commission.

This work is partly sponsored by the US Department of Energy, Office of Fusion Energy Sciences under grant number DE-SC0014229.

\section{References}

[1] Könies A 2007 (10th IAEA TM on Energetic Particles in Magnetic Confinement Systems (Kloster Seeon, Germany))

[2] Fehér T B 2013 Simulation of the interaction between Alfvén waves and fast particles Ph.D. thesis ErnstMoritz-Arndt-Universität Greifswald

[3] Hirvijoki E, Asunta O, Koskela T, Kurki-Suonio T, Miettunen J, Sipilä S, Snicker A and Äkäslompolo S 2014 Comput. Phys. Commun. 185 1310-1321

[4] Windisch T, Krämer-Flecken A, Velasco J L, Könies A, Nührenberg C, Grulke O, Klinger T and the W7X team 2017 Plasma Physics and Controlled Fusion 59105002

[5] Alfvén H 1942 Nature 150 405-406

[6] García-Muñoz M, Hicks N, van Voornveld R, Classen I G J, Bilato R, Bobkov V, Bruedgam M, Fahrbach H U, Igochine V, Jaemsae S, Maraschek M, Sassenberg K and Team A U 2010 Physical Review Letters 104185002

[7] Duong H H, Heidbrink W W, Strait E J, Petrie T W, Lee R, Moyer R A and Watkins J G 1993 Nuclear Fusion 33 749-765

[8] Darrow D S, Zweben S J, Batha S, Budny R V, Bush C E, Chang Z, Cheng C Z, Duong H H, Fang J, Fisch N J, Fischer R, Fredrickson E D, Fu G Y, Heeter R F, Heidbrink W W, Herrmann H W, Herrmann M C, Hill K, Jaeger E F, James R, Majeski R, Medley S S, Murakami M, Petrov M, Phillips C K, Redi M H, Ruskov E, Spong D A, Strait E J, Taylor G, White R B, Wilson J R, Wong K L and Zarnstorff M C 1996 Physics of Plasmas 3 1875-1880

[9] Äkäslompolo S, Wolf R, Bozhenkov S, Seki R, Isobe M, Ogawa K and Suzuki Y 2019 P2-23 (16th IAEA TM on Energetic Particles in Magnetic Confinement Systems - Theory of Plasma Instabilities (Shizuoka City, Japan))

[10] Drevlak M, Geiger J, Helander P and Turkin Y 2014 Nuclear Fusion 54073002

[11] Könies A, Mishchenko A and Hatzky R 2008 Theory of Fusion Plasmas vol 1069 ed Garbet X, Sauter O and Sindoni E (New York: American Institute of Physics) pp 133-143

[12] Garcia-Munoz M, Sharapov S E, M A Van Zeeland, Ascasibar E, Cappa A, Chen L, Ferreira J, GaldonQuiroga J, Geiger B, Gonzales-Martin J, Heidbrink W W, Johnson T, Ph Lauber, Mantsinen M, Melnikov A V, Nabais F, Rivero-Rodriguez J F, Sanchis-Sanchez L, Schneider P, Stober J, Suttrop W, Todo Y, Vallejos P, Zonca F and the AUG and MST1 Teams 2019 Plasma Physics and Controlled Fusion 61 054007

[13] Rahbarnia K, Andreeva T, Bluhm T, Borchardt M, Brandt C, Carvalho B B, Hirsch M, Kleiber R, Könies A, Laqua H P, Neuner U, Schilling J, Slaby C, v Stechow A, Thomsen H, Turkin Y, Zanini M, Zilker M and the Wendelstein 7-X Team 2019 P2.1066 (46th EPS Conference on Plasma Physics (Milan, Italy))

[14] Grieger G, Lotz W, Merkel P, Nührenberg J, Sapper J, Sturmberger E, Wobig H, the W7-X Team, Burhenn 
R, Erckmann V, Gasparino U, Giannone L, Hartfuss H J, Jaenicke R, Kühner G, Ringler H, Weller A, Wagner F and the W7-AS Team 1992 Physics of Fluids B 42081

[15] Erckmann V, Hartfuß H J, Kick M, Renner H, Sapper J, Schauer F, Speth E, Wesner F, Wagner F, Wanner M, Weller A, Wobig H, the W7-AS team and the W7-X teams at IPP Garching, FZK Karlsruhe and IPF Stuttgart 1998 17th IEEE/NPSS Symposium Fusion Engineering (Cat. No.97CH36131) vol 1 (San Diego, CA, USA: IEEE) pp 40-48 ISBN 978-0-7803-4226-2 URL http://ieeexplore. ieee.org/document/ 685662/

[16] Klinger T et al 2019 Nuclear Fusion 59112004

[17] Du X D, Toi K, Osakabe M, Ohdachi S, Ido T, Tanaka K, Yokoyama M, Yoshinuma M, Ogawa K, Watanabe K Y, Isobe M, Nagaoka K, Ozaki T, Sakakibara S, Seki R, Shimizu A, Suzuki Y, Tsuchiya H and LHD Experiment Group 2015 Physical Review Letters 114155003

[18] Heidbrink W W, Bardoczi L, Collins C S, Kramer G J, R J La Haye, Lin D J, Muscatello C M, Podestà M, Stagner L, M A Van Zeeland and Zhu Y B 2018 Nuclear Fusion 58082027

[19] Kleiber $\mathrm{R}$ et al 2019 private communications (paper in preparation)

[20] Li Y, Liu K J R and Razavilar J 1995 Digital Repository at the University of Maryland T.R.95-11

[21] Li Y, Razavilar J and Liu K J R 1998 IEEE Transactions on Biomedical Engineering 45 78-86

[22] Peeters B and De Roeck G 2001 Journal of Dynamic Systems, Measurements, and Control 123 659-667

[23] Magalhães F and Cunha Á 2011 Mechanical Systems and Signal Processing 25 1431-1450

[24] Brandt C, Schilling J, Thomsen H, Broszat T, Laube R, Schröder T, Andreeva T, Beurskens M N A, Bozhenkov S A, Brunner K J, Card A, Cordes C, Damm H, Fuchert G, Gallowski K, Gutzmann R, Knauer J, Laqua H P, Marquardt M, Ph Nelde, Neuner U, Pasch E, Rahbarnia K, Recknagel J, Schülke M, Scott E R, Sieber T and the W7-X Team 2020 Plasma Physics and Controlled Fusion 62035010

[25] Könies A and Eremin D 2010 Physics of Plasmas 17012107

[26] Slaby C, Könies A, Kleiber R and García-Regaña J M 2018 Nuclear Fusion 58082018

[27] Slaby C, Könies A, Kleiber R, Äkäslompolo S and Kontula J 2018 Journal of Physics: Conference Series 1125012019

[28] Slaby C, Könies A, Kleiber R and Leyh H 2019 Nuclear Fusion 59046006

[29] Slaby C, Könies A, Kleiber R, Äkäslompolo S and Kontula J 2018 Europhysics Conference Abstracts 42A P4.1098

[30] van Overschee P and De Moor B 1996 Subspace Identification for Linear Systems (Kluwer Academic Publishers Boston/London/Dordrecht)

[31] Turkin Y, Beidler C D, Maaßberg H, Murakami S, Tribaldos V and Wakasa A 2011 Physics of Plasmas 18 022505

[32] Hirshman S P and Whitson J C 1983 The Physics of Fluids 26 3553-3568

[33] Chu M S, Greene J M, Lao L L, Turnbull A D and Chance M S 1992 Physics of Fluids B: Plasma Physics $43713-3721$

[34] Schwab C 1993 Physics of Fluids B: Plasma Physics 5 3195-3206

[35] Helander P and Simakov A N 2008 Physical Review Letters 101145003

[36] Helander P 2014 Reports on Progress in Physics 77087001

[37] Dinklage A, Beidler C D, Helander P, Fuchert G, Maaßberg H, Rahbarnia K, Sunn Pedersen T, Turkin Y, Wolf R C, Alonso A, Andreeva T, Blackwell B, Bozhenkov S, Buttenschön B, Czarnecka A, Effenberg F, Feng Y, Geiger J, Hirsch M, Höfel U, Jakubowski M, Klinger T, Knauer J, Kocsis G, Krämer-Flecken A, Kubkowska M, Langenberg A, Laqua H P, Marushchenko N, Mollén A, Neuner U, Niemann H, Pasch E, Pablant N, Rudischhauser L, Smith H M, Schmitz O, Stange T, Szepesi T, Weir G, Windisch T, Wurden G A, Zhang D and the W7-X Team 2018 Nature Physics 14 855-860

[38] Helander P and Sigmar D J 2002 Collisional Transport in Magnetized Plasmas (Cambridge University Press)

[39] Zanini M, Laqua H P, Stange T, Brandt C, Damm H, Hirsch M, Höfel U, Rahbarnia K, Strumberger E, Thomsen H, Wolf R C and the W7-X Team 2019 P5.1058 (46th EPS Conference on Plasma Physics (Milan, Italy))

[40] Äkäslompolo S, Drewelow P, Gao Y, Ali A, Asunta O, Bozhenkov S, Fellinger J, Ford O P, N den Harder, Hartmann D, Jakubowski M, McNeely P, Niemann H, Pisano F, Rust N, A Puig Sitjes, Sleczka M, Spanier A, Wolf R C and the W7-X Team 2019 Journal of Instrumentation 14 P07018

[41] Äkäslompolo S, Drewelow P, Gao Y, Ali A, Bozhenkov S, Fellinger J, Geiger J, Hartmann D, Hathiramani D, Jakubowski M, McNeely P, Mohr S, Niemann H, Pisano F, Rust N, Sitjes A P, Sleczka M, Wolf R and W7-X Team 2019 Fusion Engineering and Design 146 862-865 